\title{
A molecular map of murine lymph node blood vascular endothelium at single cell resolution
}

\author{
Kevin Brulois 1,13, Anusha Rajaraman1,2,3,13, Agata Szade (1) 1,4,13, Sofia Nordling 1,13, Ania Bogoslowski (1) 5,6, \\ Denis Dermadi (1) ${ }^{1}$, Milladur Rahman (1) 1, Helena Kiefel ${ }^{1}$, Edward O'Hara', Jasper J. Koning ${ }^{3}$, Hiroto Kawashima ${ }^{7}$, \\ Bin Zhou (1) ${ }^{8}$, Dietmar Vestweber (10 ${ }^{9}$, Kristy Red-Horse ${ }^{10}$, Reina E. Mebius ${ }^{3}$, Ralf H. Adams (iD ${ }^{11}$, \\ Paul Kubes (10) ${ }^{5,6}$, Junliang Pan ${ }^{1,2} \&$ Eugene C. Butcher ${ }^{1,2,12 凶}$
}

Blood vascular endothelial cells (BECs) control the immune response by regulating blood flow and immune cell recruitment in lymphoid tissues. However, the diversity of BEC and their origins during immune angiogenesis remain unclear. Here we profile transcriptomes of BEC from peripheral lymph nodes and map phenotypes to the vasculature. We identify multiple subsets, including a medullary venous population whose gene signature predicts a selective role in myeloid cell (vs lymphocyte) recruitment to the medulla, confirmed by videomicroscopy. We define five capillary subsets, including a capillary resident precursor (CRP) that displays stem cell and migratory gene signatures, and contributes to homeostatic BEC turnover and to neogenesis of high endothelium after immunization. Cell alignments show retention of developmental programs along trajectories from CRP to mature venous and arterial populations. Our single cell atlas provides a molecular roadmap of the lymph node blood vasculature and defines subset specialization for leukocyte recruitment and vascular homeostasis.

\footnotetext{
${ }^{1}$ Laboratory of Immunology and Vascular Biology, Department of Pathology, Stanford University School of Medicine, Stanford, CA, USA. ${ }^{2}$ Palo Alto Veterans Institute for Research, Palo Alto, CA, USA. ${ }^{3}$ Department of Molecular Cell Biology and Immunology, Vrije Universiteit Medical Center, Amsterdam, The Netherlands. ${ }^{4}$ Department of Medical Biotechnology, Faculty of Biochemistry, Biophysics and Biotechnology, Jagiellonian University, Krakow, Poland. ${ }^{5}$ Calvin, Phoebe \& Joan Snyder Institute for Chronic Diseases, Cumming School of Medicine, University of Calgary, Calgary, AB T2N 4N1, Canada. ${ }^{6}$ Department of Physiology and Pharmacology, Cumming School of Medicine, University of Calgary, Calgary, AB T2N 4N1, Canada. ${ }^{7}$ Department of Biochemistry, School of Pharmacy and Pharmaceutical Sciences, Hoshi University, Tokyo, Japan. ${ }^{8}$ The State Key Laboratory of Cell Biology, CAS Center for Excellence in Molecular Cell Science, Shanghai Institute of Biochemistry and Cell Biology, Chinese Academy of Sciences, 200031 Beijing, China. ${ }^{9}$ Department Vascular Cell Biology, Max Planck Institute for Molecular Biomedicine, Münster, Germany. ${ }^{10}$ Department of Biology, Stanford University, Stanford, CA, USA. ${ }^{11}$ Max Planck Institute for Molecular Biomedicine, Department of Tissue Morphogenesis, University of Münster, Faculty of Medicine, Münster, Germany.

12 The Center for Molecular Biology and Medicine, Veterans Affairs Palo Alto Health Care System, Palo Alto, CA, USA. ${ }^{13}$ These authors contributed equally:

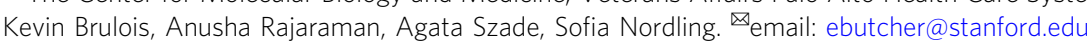


T he vascular endothelium lining blood vessels regulates exchange of oxygen and metabolites between the blood vascular compartment and tissues. In lymph nodes (LN), additionally, the endothelium plays essential roles in controlling immune cell access. The organization of the vasculature in $\mathrm{LN}$ is well characterized: Arteries entering at the hilus lead to capillary arcades in the LN cortex; capillaries link to 'high endothelial venules' (HEV), the post capillary venules that recruit lymphocytes from the blood ${ }^{1}$; and the vasculature exits the lymph node at the hilus. Upon immunization, lymph nodes can increase in volume 10-fold or more within days, and the vascular endothelium expands roughly proportionally from local precursors ${ }^{1,2}$. Vessel expansion involves extensive proliferation of both capillary and high endothelial cells (HEC) ${ }^{3}$, without contribution from blood borne progenitors ${ }^{4}$; and an increase in vessel numbers through intussusceptive (splitting) angiogenesis ${ }^{5}$. However, the nature and extent of endothelial cell diversity within LN remains incompletely understood.

Single-cell RNA profiling is a transformative technology for the identification of cell diversity and elucidation of developmental and physical relationships. Here we provide a survey of blood vessel endothelial cells (BEC) from mouse peripheral lymph nodes (PLN). We confirm known features of high endothelium ${ }^{6,7}$, identify endothelial subsets, describe unexpected diversity among capillary cells and demonstrate a distinctive role of medullary veins in selective myeloid cell recruitment. We define gene signatures and transcription regulatory factors for these subsets, and map key subsets to the vasculature. We also identify a primed capillary resident regenerative population (CRP) that displays the angiogenic endothelial marker Apln, is enriched in cells undergoing cell division, and possesses stem cell and migratory gene signatures. Genetic lineage tracing suggests that CRP function as progenitors, contributing to neogenesis of the blood vascular endothelium including high endothelium in immune angiogenesis.

\section{Results}

Single-cell profiling of lymph node blood endothelial cells. We performed single-cell RNA sequencing (scRNAseq, 10× Chromium) of sorted BEC from PLN of adult mice (Fig. 1a). Four cohorts were analyzed including a group of male and female $\mathrm{Balb} / \mathrm{c}$ mice (PLN1) processed together and resolved in silico (PLN1_m and PLN1_f), an additional group of female Balb/c mice (PLN2), and one of female mice of a mixed background (PLN3) (Supplementary Fig. 1a). Unsupervised analyses (Methods) defined 8 BEC subsets with distinct gene expression: arterial EC (Art); 2 venous EC subsets, high endothelial cells (HEC) and non-HEC veins $(\mathrm{Vn})$; and five capillary phenotype EC subsets, including a transitional phenotype capillary EC (TrEC) and primed EC comprising a capillary resident regenerative population (CRP) (Fig. 1b-d). Annotations of the arterial and venous populations were guided by known gene markers ${ }^{6,8,9}$. Nearest neighbor alignments, which can model developmental relationships or spatial alignments of cells, were visualized by trajectory inference using $\mathrm{tSpace}^{10}$, which revealed a continuum of cell phenotypes with branching of arterial and venous subsets from capillary EC populations (Fig. 1e). Clusters extending to the termini of the arterial, CRP, and HEV branches were further separated to distinguish cells that were most distinct (distant along trajectories) from the bulk of EC (darker shading, Fig. 1e): these cells are enriched for genes associated with mature arterial (e.g., Gkn3, Bmx) or HEV (Chst4, Glycam1) differentiation, or in the case of CRP, for genes associated with endothelial specification during early development or with stem cells (e.g., Ets2, Cxcr4, $\mathrm{Nes}$ (Fig. 1f). Clusters and trajectory alignments were shared by male and female mice and by the independently processed samples (Supplementary Fig. 1b-d). Gene expression signatures were generated for each subset using the combined scRNAseq datasets (Methods; Supplementary Data 1). Correlation in mean gene expression profiles of the identified subsets across the cohorts is shown in Supplementary Fig. 1e. Expression of marker genes depicted in Fig. If showed overall consistency across all biological replicates (Supplementary Fig. 2).

Characterization of the arterial and venous subsets. The arterial cluster among the profiled BEC was identified by expression of Gja5 and Gja4 (encoding connexin 37 and 40, respectively) and $B m x$ (Fig. 1f). Consistent with prior reports describing Gkn3 as a marker for mature arteries ${ }^{8}$, it is selectively expressed in mature Art, and absent in pre-Art, which lay closer to the capillary subsets in trajectory space (Fig. 1e). In contrast, Depp1 is preferentially expressed in pre-Art, consistent with a prior study showing its transient expression in developing arterial endothelial cells and subsequent downregulation in mature vessels ${ }^{11}$ (Fig. 1f). Pre-Art and Art express Klf2 and Klf4, genes induced by laminar shear flow and preferentially associated with linear segments compared to branched vessels ${ }^{9,12}$.

Venous EC, comprising HEC and non-HEC (Vn) subsets, share expression of the vein-specifying transcription factor $\mathrm{Nr} 2 \mathrm{f2}$ (Coup-TFII $)^{13}$, and the vein-associated chemokine interceptor Ackr1 (DARC ${ }^{14}$; Fig. 1f). HEC express genes required for lymphocyte recruitment including Chst 4 and Glycam $1^{15}$, with more pronounced expression on HEC distal in tSpace (late HEC; Fig. 1e, f). Consistent with their large size and plump morphology and with prior whole-genome expression studies of sorted $\mathrm{HEC}^{6}$, their gene signature is enriched for glycoprotein synthesis (Supplementary Data 1), and they have uniquely high numbers of transcripts per cell (Fig. 1g).

Cells of the Vn subset branch prominently from proximal HEC and TrEC in tSpace projection (Fig. 1e). To identify these cells in the LN vasculature, we imaged whole LN removed shortly after i.v. delivery of fluorescently labeled antibodies (Methods): antiLy6c, specific for arteries and capillaries; MECA79 to the Peripheral Node vascular Addressin (PNAd) defining HEV; and anti-PLVAP, which stains capillary and venous EC but not arteries (Fig. 2a). Subset markers allow visualization of arterial entry from the LN hilus, linking to capillary arbors in the cortex which in turn connect to HEC. We identified PNAd ${ }^{-}$veins downstream of and as a continuation of HEV in the lymph node medulla (Fig. 2a): they bound injected antibodies to VE-cadherin, PLVAP, and ICAM1 (Fig. 2b) but were negative for capillary markers Ly6c and podocalyxin (PODXL; Supplementary Fig. 4), as predicted by gene expression (Fig. 2c).

The $\mathrm{Vn}$ gene signature includes genes associated with regulation of neutrophil activation (GO:1902563; Fig. 3a) and platelet degranulation (GO:0002576; Supplementary Fig. 3). Vn express Von Willebrand Factor $(V w f)$, which is stored in WeibelPalade bodies and released during inflammation to promote platelet adhesion and hemostasis ${ }^{16}$. Surprisingly, Vn lack HEC-associated genes for naïve lymphocyte recruitment (Chst4, Fut7, Ccl21), instead expressing genes for vascular E- and Pselectins (Sele and Selp) and adhesion receptors ICAMland VCAM1 (Fig. 2c), which mediate myeloid cell recruitment. Neutrophils and monocytes are normally excluded from LN homing, but they enter LN's in large numbers in response to acute inflammation and play an important role in preventing pathogen spread. Prior studies have characterized inflammatory changes in HEC, which enable recruitment of myeloid cells along with lymphocytes ${ }^{17-19}$; but the role of medullary Vn has not been examined. We induced inflammation by footpad injection of the 
a

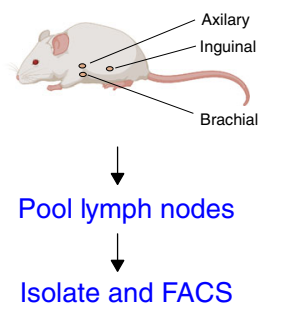

blood endothelial cells (BEC)

C

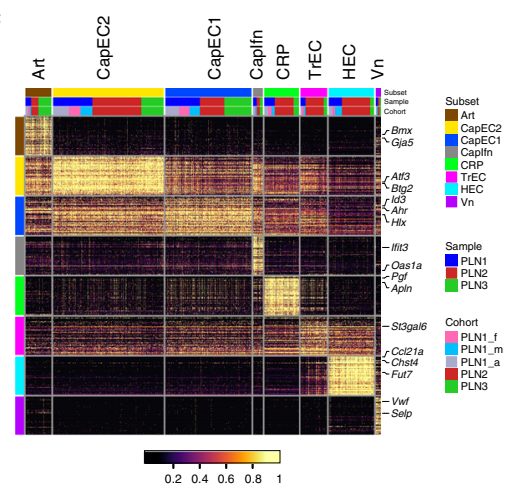

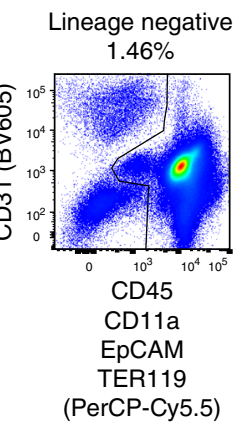

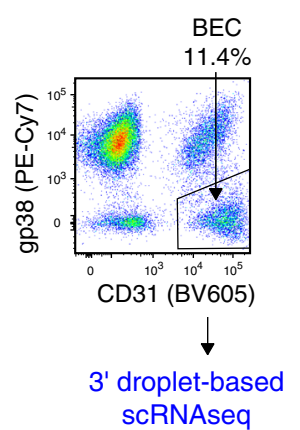

d

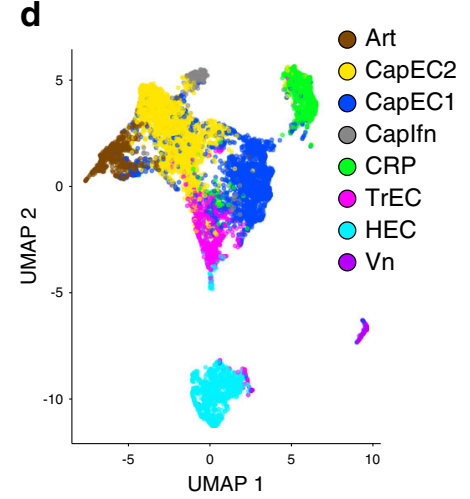

b

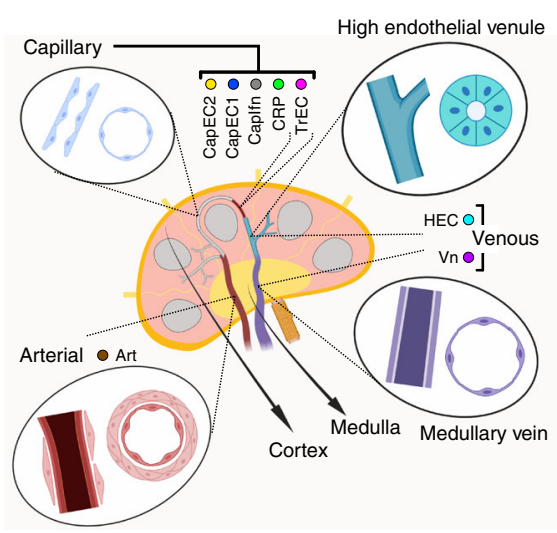

e

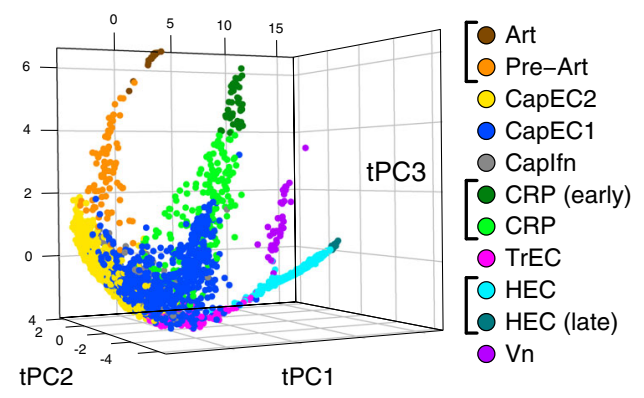

tPC2

g

$\mathbf{f}$
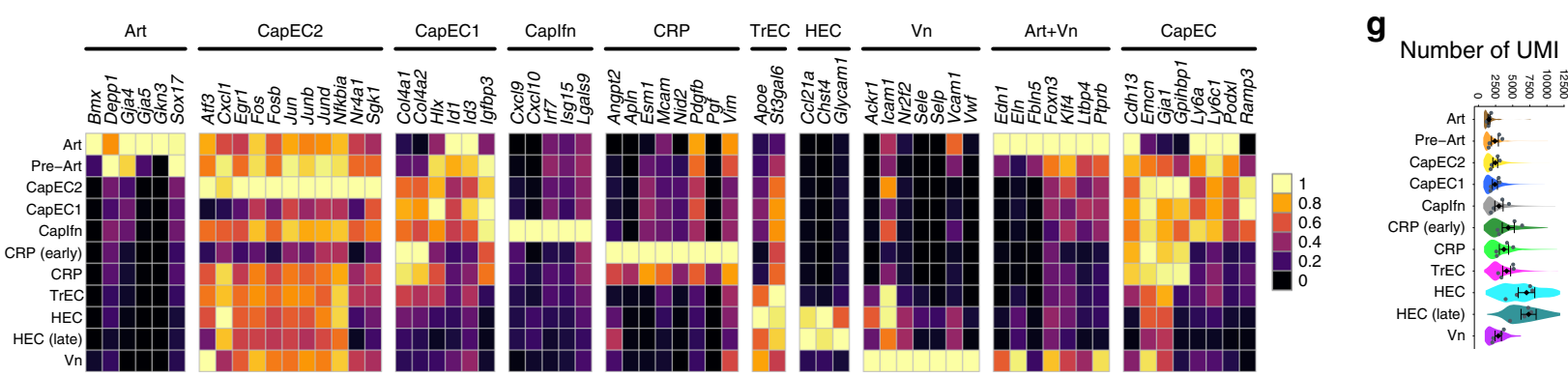

Fig. 1 Single-cell survey of lymph node blood vessel endothelial cells. a Workflow schematic. Lymph nodes from adult mice are pooled and dissociated into single cells. Fluorescence activated cell sorting (FACS) is used to isolate blood endothelial cells. scRNAseq ( $\times 10$ Chromium) is used to profile the cells. Representative FACS plot. $\mathbf{b}$ Lymph node schematic depicting the eight major subsets identified by scRNAseq analysis. $\mathbf{c}$ Heatmap of expression of the top 50 differentially and specifically expressed genes for each subset are shown. Subset, sample, and cohort are annotated across the top and select genes on the right. d UMAP plot of 8832 single cells from three samples (four cohorts). Cells are segregated by type: arterial EC (pre-Art), high endothelial cells $(\mathrm{HEC})$, non-HEC veins $(\mathrm{Vn})$, and five capillary phenotype EC (CapEC1, CapEC2, capillary resident progenitors (CRP), transitional EC (TrEC), Interferonstimulated gene-enriched CapEC (Caplfn). e Computationally predicted relationships visualized in PCA projection of cells aligned in trajectory space using cells from PLN1. Clusters extending to the termini of the arterial, CRP, and HEV branches were further subdivided to distinguish cells most distinct (distant along trajectories) from the bulk of EC (CRP (early), Art, HEC (late; darker shadings). Interactive rendering available: https://stanford.io/2qzJ8HI.

f Selected marker and signature genes for each of the indicated clusters and combinations of clusters (top). Mean expression values from three samples and four cohorts (color scale). $\mathbf{g}$ Total transcript counts (UMl; unique molecular identifiers) per cell within each subset. Violins show the UMI distribution of all cells. Mean expression values for each of the four independent cohorts (gray dots) and mean and standard error (SEM) of the cohort means are also plotted (black diamonds, bars).

bacterial pathogen S. aureus in mice with green fluorescent protein (GFP) expressing neutrophils (LysM $\mathrm{MFP}^{\mathrm{GF}}$ mice). One hour later, mice were transfused with CMTPX-labeled lymphocytes and recruitment was quantified by live two-photon imaging of the popliteal LN. PNAd ${ }^{-}$medullary venules exhibited massive and exclusive recruitment of $\mathrm{GFP}^{+}$myeloid cells, contrasting with both lymphocyte and induced myeloid cell interactions in HEV (Fig. 3b-e). In contrast to recruitment through HEV which is PNAd-dependent ${ }^{18}$, myeloid recruitment was robust even in the presence of blocking concentrations of anti-PNAd. Anti-Pselectin significantly inhibited $\mathrm{GFP}^{+}$cell accumulation on Vn, and combined inhibition of vascular E- and P-selectins largely abrogated medullary vein interactions of myeloid cells (Fig. 3f), while HEV recruitment was unaffected ${ }^{18}$. Thus venules in the medullary environment have a unique phenotype and function, selectively recruiting myeloid cells to the medulla in acute inflammation. The results illustrate a surprisingly localized BEC programming for differential leukocyte recruitment. 

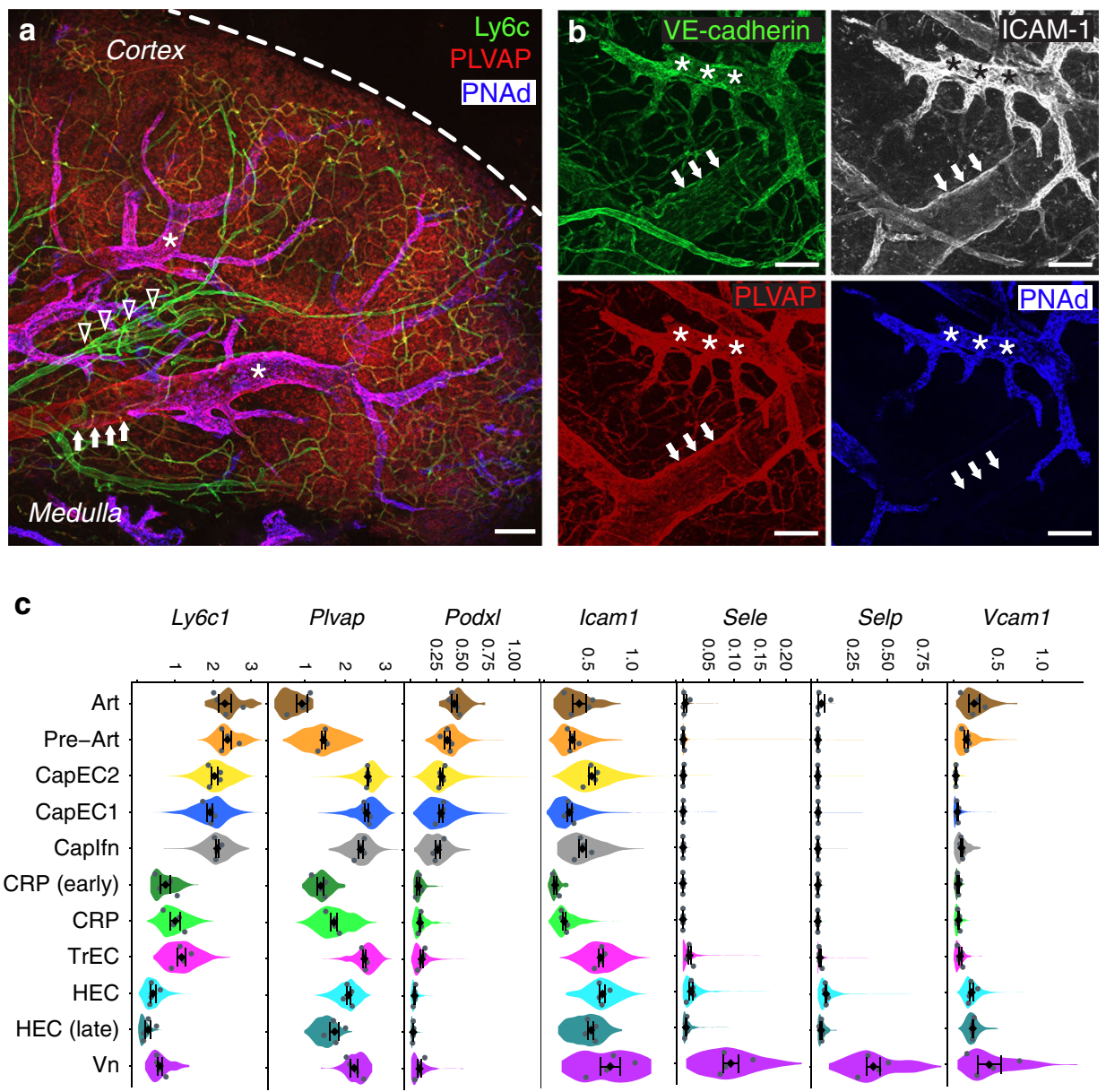

Fig. 2 Marker gene expression and immunolocalization of the major arterial and venous populations. Immunofluorescent visualization of PLN vessels using intravenously (i.v.) injected antibodies: anti-Ly6c (green), anti-PLVAP (red), and anti-PNAd (blue). Dashed line, lymph node capsule (a); anti-VEcadherin (green), anti-ICAM-1 (white), anti-PLVAP (red), and anti-PNAd (blue) (b). Arrow heads, artery (Ly6 $\mathrm{C}^{+}$PNAd $^{-}, \mathrm{PLVAP}^{-}, \mathrm{ICAM}^{\mathrm{low}}$ ). Arrows, medullary veins $\left(\mathrm{PNAd}^{-}, \mathrm{ICAM}^{+}, \mathrm{PLVAP}^{+}, \mathrm{Ly}_{6} \mathrm{C}^{-}\right.$). Medullary veins are downstream of HEC. Asterisks, HEV (PNAd $\left.{ }^{+}\right)$. Bars, $100 \mu \mathrm{m}$. Dotted line, medullary vein. Images representative of three independent experiments. c Violin plots showing expression of genes Ly6c1, Plvap, Podxl, and Icam1 corresponding to immuno-stained marker proteins; and Sele, Selp and Vcam1 illustrating selective expression by non-HEV vein. Note the decline in Podxl expression from artery to pre-Art to capillary EC subsets, and a corresponding decline in intensity of staining for Ly6c1 as arteries bifurcate into capillaries in situ in a. Mean expression values for each of the four independent cohorts (grey dots) and mean and SEM of the cohort means are also plotted (black diamonds) within the violin plots.

Characterization of capillary subsets. Five capillary populations shared expression of Cdh13, Emcn, Gja1, and Gpihbp1, previously described as gene markers of capillary EC in $\mathrm{PLN}^{6}$ (Fig. 1f). A transitional phenotype subset (TrEC) expresses canonical capillary genes as well as some HEC genes, albeit at low levels compared to bona fide HEC (Fig. 4a, b). TrEC bridge other CapEC to the venous branch in tSpace projections (Fig. 1e), further suggesting a close relation to HEC. They express Chst2 and the HEC genes St3Gal6 and Fut7, which encode glycosyltransferases for the synthesis of sialyl LewisX (SLex) and 6-sulfo-SLex, carbohydrates that can initiate tethering of lymphocytes under shear flow ${ }^{15}$. Synthesis of PNAd, the mature multivalent L-selectin ligand for lymphocyte homing that defines HEV also involves Chst 4 and requires the core 2-branching enzyme encoded by Gcnt1. Chst 4 and Gcnt1 are nearly undetectable in TrECs, suggesting that TrEC and HEC might display different glycotopes. Thus, we used antibodies to SLex and PNAd to identify TrEC in situ. Imaging revealed a significant population of BEC that co-stained for SLex and for capillary antigens (Fig. 4c, Supplementary Fig. 5) but lacked mature PNAd. They were morphologically thin-walled and were found immediately upstream of $\mathrm{HEV}$, correlating with their position in trajectory space (Fig. 1e).

CapEC1 and CapEC2, the two most abundant capillary populations (Fig. 1c), are centrally located among EC in trajectory space, acting as a hub from which differentiated venous and arterial branches emerge (Fig. 1e). The CapEC1 gene signature is enriched for Type IV collagen trimers (GO:0005587; Col4a1, Col4a2) as well as $I g f b p 3$ involved in maintaining vascular tone and blood pressure ${ }^{20}$. They have high vascular endothelial growth factor receptor activity (GO:0005021), yet also express negative regulators of angiogenesis ( $H l x$ and $I g f b p 3)$ and have enhanced expression of genes $I d 1$ and $I d 3$ encoding inhibitor of differentiation proteins, bHLH transcriptional regulators that restrain cell differentiation and delay cellular senescence $e^{21,22}$ (Fig. 1f).

CapEC2 express Egr1, Cxcl1 and genes reflecting NFkB activation (Nfkbia, Nfkbid, Nfkbiz) and JNK activation (Jun, Junb, Jund, Fos, Fosb, Atf3). These genes and pathways are induced by spatial gradients in fluid shear stress ${ }^{23}-25$. Compared to CapEC1, CapEC2 also show reduced Gja4 and Id1 and 
a GO:0048247 lymphocyte chemotaxis

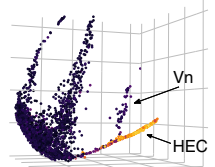

C

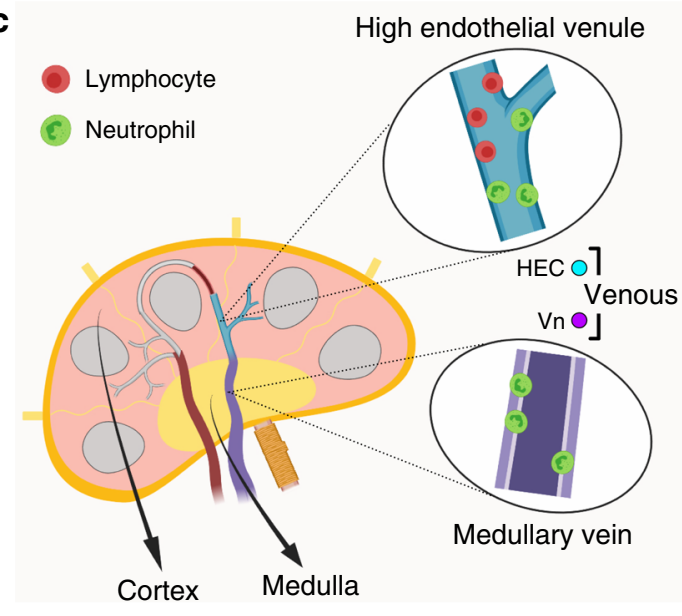
regulation of neutrophil activation

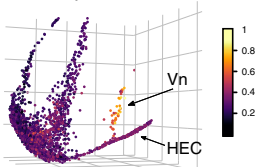

b
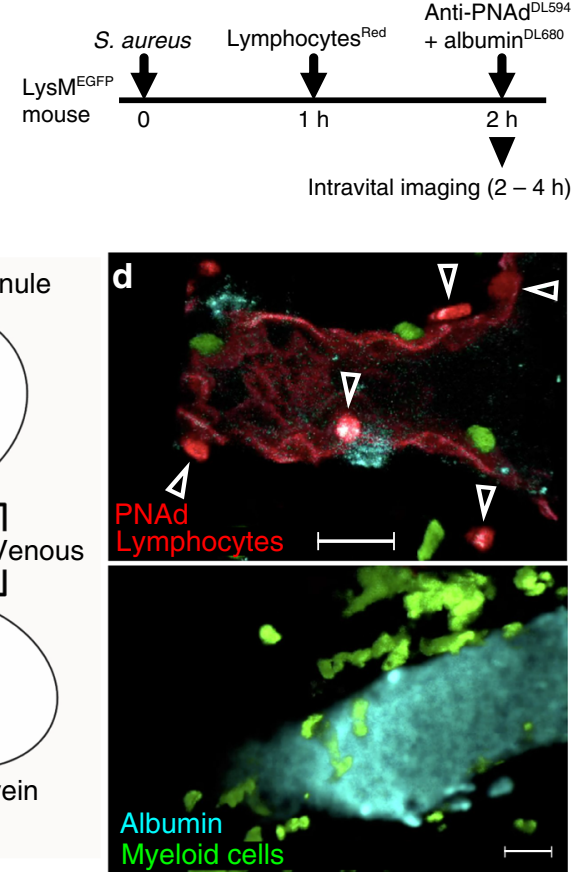

e

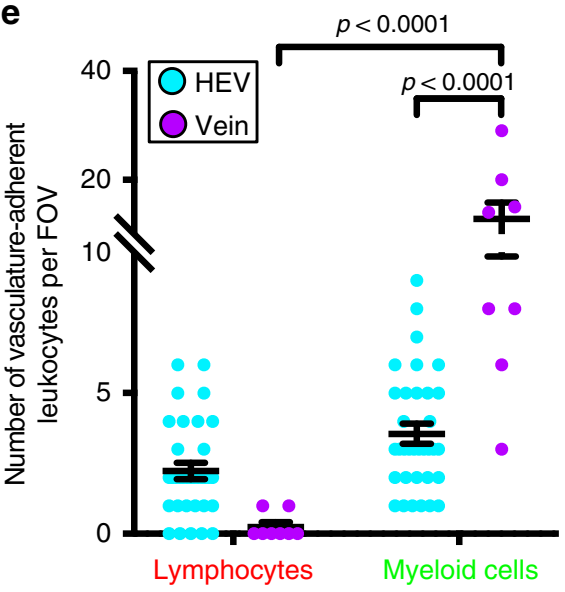

f

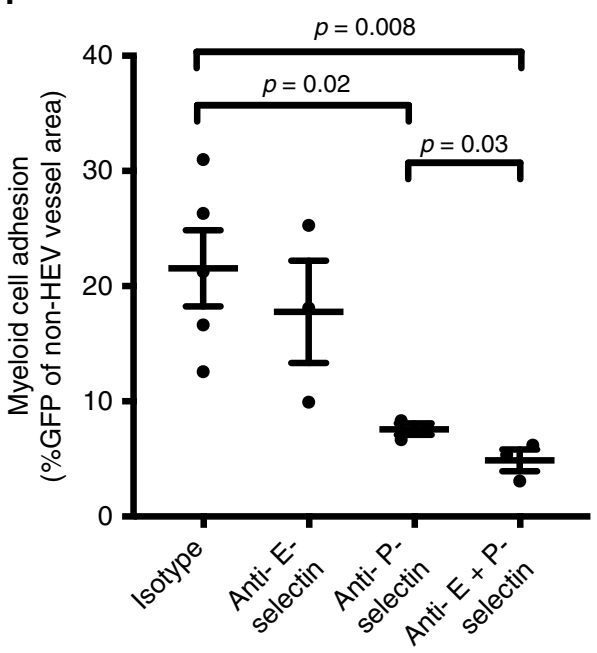

Fig. 3 Medullary veins recruit myeloid cells but not lymphocytes in acute inflammation. In situ lymphocyte and myeloid cell recruitment in HEV versus medullary veins of $\mathrm{S}$. aureus infected LysMGFP mice. a Pooled expression values of genes from the indicated GO terms (color scale) plotted along the tSpace projection from Fig. 1e. $\mathbf{b}$ Experimental protocol for S. aureus injection and visualization of lymphocyte and myeloid cell trafficking in LN. LysM ${ }^{\text {GFP }}$ recipients received $2.5 \times 10^{7} \mathrm{~S}$. aureus in the footpad. One hour later mice were injected i.v. with CMTPX-labeled lymphocytes (red). The draining LN was imaged from 2-4 h post infection using two-photon videomicroscopy. c Schematic depicting the location of HEV and medullary veins visualized.

d Representative fields of view from 2 photon videomicroscopy of a LN from mice treated according to (b). Myeloid cells (green) and lymphocytes (red; arrow heads) arrested in HEV (upper panel) or medullary vein (lower panel). HEV, identified by injection of red fluorescent anti-PNAd at a non-blocking concentration immediately prior to sacrifice, are readily distinguished from migrating lymphocytes and from PNAd ${ }^{-}$medullary veins. Venular lumen is highlighted by Dylight-680 labeled albumin (cyan). Bars, 20 um. e Quantification of lymphocyte and myeloid cells adherent to HEV and medullary veins. $n=34$ fields of view (FOV) for HEV and 8 FOV for veins from a total of four mice. Data shown as mean \pm SEM. Statistically significant differences were determined using a two-way ANOVA test corrected with Tukey. $\mathbf{f}$ Inhibition of myeloid cell accumulation in medullary veins by antibody blockade of P- and $\mathrm{E}$ - selectin. Test or isotype control antibodies were injected i.v. 20 min before footpad S. aureus infection in LysMGFP mice, and draining LN visualized $2 \mathrm{~h}$ post infection. Myeloid cell $\left(\mathrm{GFP}^{+}\right)$adhesion to medullary veins was quantified from 24-47 FOV of popliteal LN over $2 \mathrm{~h}$ of imaging. Each point represents an average of the values collected from one mouse. $n=5$ mice for isotype, $n=3$ mice for all other groups. Data shown as mean \pm SEM. A one-tailed $t$-test was used for comparisons to the Isotype. A two-tailed $t$-test was used to compare Anti-P-selectin and Anti-E + P-selectin. 

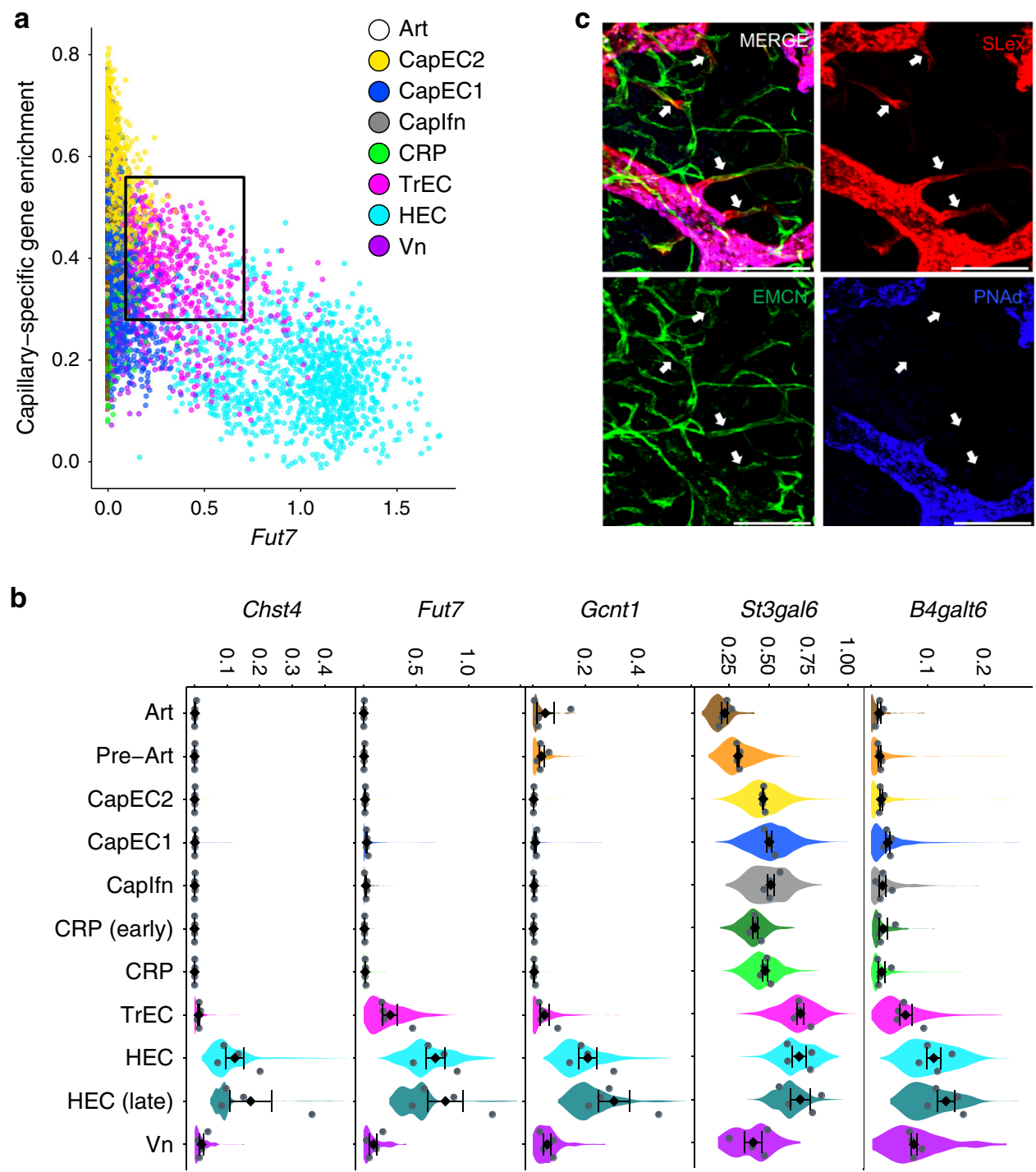

Fig. 4 Transitional phenotype capillary EC occupy capillary-HEC junctions. a Scatter plot of cells showing Fut7 expression by capillary EC defined by an enrichment score for capillary-specific genes. Cells colored by major cell type. b Immunofluorescence image of PLN with intravenously injected anti-SLex (red), anti-PNAd (blue), and anti-capillary (EMCN; green) antibodies. Scale bar $100 \mu \mathrm{m}$. Arrows point to Slex ${ }^{+} \mathrm{EMCN}^{+} \mathrm{PNAd}^{-} \mathrm{TrEC}^{-}$Images representative of three independent experiments. c Expression of Chst2, Fut7, Gcnt1, St3gal6, B4galt6 in the BEC subsets. Violins show the expression distribution of all cells. Mean expression values for each of the four independent cohorts (grey dots) and mean and SEM of the cohort means are also plotted (black diamonds).

enrichment for genes in the MAPK cascade (GO:0000165) and WNT signaling (GO:0060070; Supplementary Fig. 3), characteristics that parallel findings from a ChIP-seq-based analysis of a disturbed oscillatory shear stress response ${ }^{26}$. These CapEC2 features suggest exposure to gradient or disturbed shear stress.

A third relatively rare population, CapIfn, has a prominent signature of interferon signaling (GO:0060337), with high expression of transcription factor Irf7, interferon response genes Ifit1, Ifit2, and chemokines CXCL9 and 10 (Fig. 1f). CapIfn also express Isg15 and Gadd45a, genes associated with an "apoptotic-like" signature described in other single-cell studies ${ }^{27}$.

An angiogenic capillary subset enriched for stem-cellassociated genes. Unsupervised clustering identified a distinctive population of activated capillary endothelial cells, CRP, that occupy their own branch in trajectory space, linking to CapEC1, 2 and TrEC (Fig. 1e). Their gene expression signatures reveal a progenitor-like phenotype. They have uniquely high expression of Ets 2 and Sox7, encoding transcription factors implicated in early specification of endothelial cells during development and they display genes and features of stem or progenitor cells in other systems ${ }^{28}$. These developmental EC genes are additionally enriched in early vs late CRP as defined by cell positions along their $\mathrm{t}$ Space branch. CRP also express genes associated with neural and hematopoietic stem or progenitor cells including $\mathrm{CxCr} 4^{29}, \mathrm{Nes}^{30}, \mathrm{Kit}^{31}, \mathrm{Lxn}^{32}$, and Sox $4^{33}$; and they are enriched in spliceosome genes (GO:0097525) including Snrpal and Snrpd1 (Fig. 5a), which participate in the acquisition and maintenance of pluripotency in embryonic stem cells ${ }^{34}$. Like embryonic and neural stem cells, CRP uniquely lack expression of Neat1, a long noncoding gene widely expressed in differentiated cells for paraspeckle assembly and double stranded RNA processing during stem cell fate selection ${ }^{35}$ (Fig. 5a). Multipotent stem and progenitor cells have more diverse gene and protein 


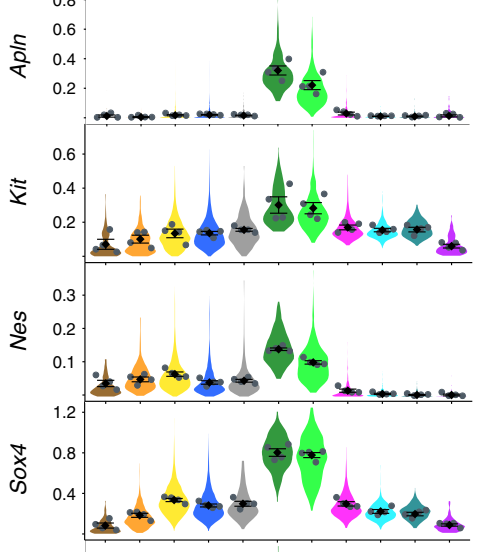

$\begin{array}{ll}0 & 0.15 \\ \text { ิิ } & 0.10 \\ \text { ป } & 0.05\end{array}$
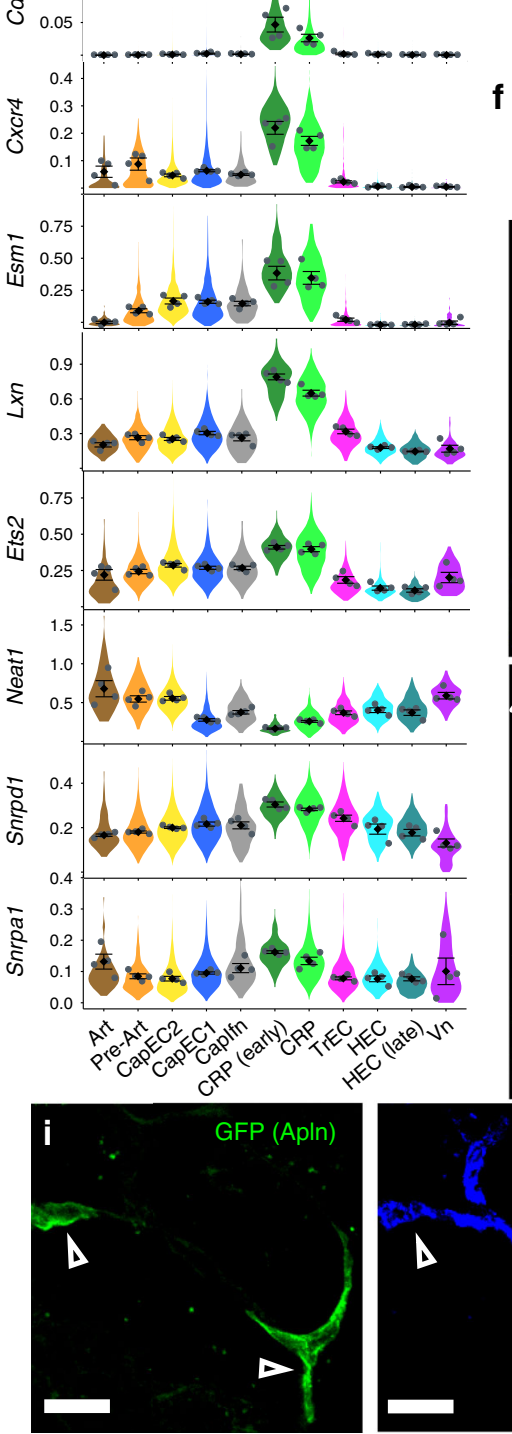

b

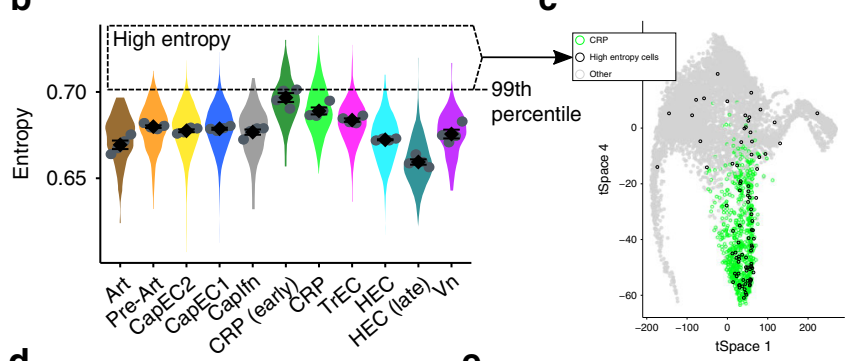

d

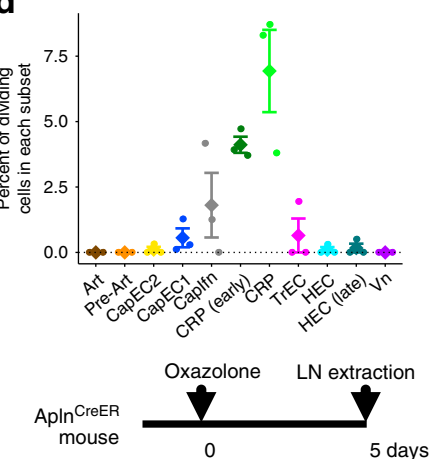

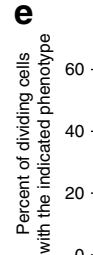

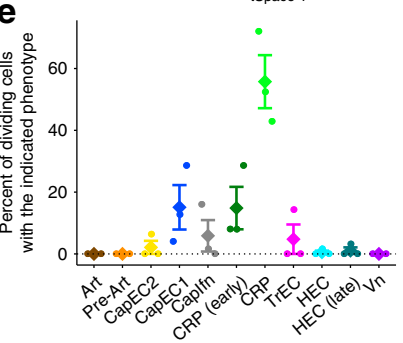

g
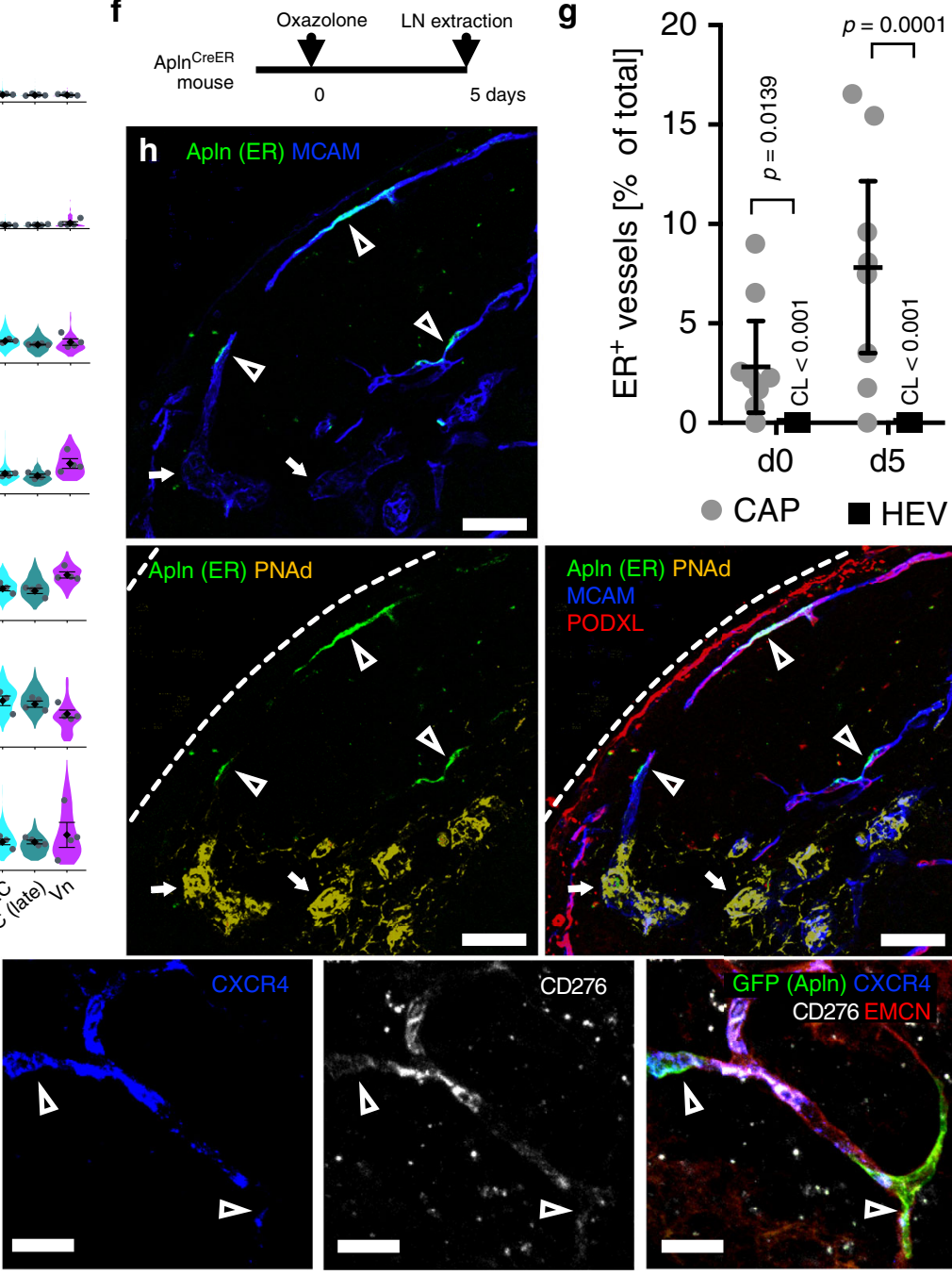

signaling than their differentiated progeny, reflecting their developmental plasticity. These characteristics can be quantified by calculating the 'signaling entropy rate' using the SCENT algorithm ${ }^{36}$. Early CRP display higher entropy than other EC subsets, while Art and late HEC subsets display the lowest entropy (Fig. 5b). Dividing cells among CapEC1 share some of these correlates of potency, including high entropy; however, cells with the highest entropy tend to map to the 'origin' of the CRP branch in trajectory space (Fig. 5c). Finally, CRP express Angpt2, Apln, Esm1, Nid2, Pdgfb, and Pgf (Fig. 1f), genes characteristic of angiogenic tip cells, precursors to new vessels formed during sprouting angiogenesis ${ }^{37,38}$. Together these observations suggest 
Fig. 5 Stem cell features, markers and immunolocalization of a capillary resident regenerative population (CRP). a Expression of selected stem or progenitor cell-related genes. b Signaling entropy rate (entropy) for each BEC subset. Highest $1 \%$ of all BEC, dashed box. High entropy cells are enriched in early CRP. c tSpace projection from Supplementary Fig. 1d with all cells. CRP, green. High Entropy cells (top 1\% as gated in (b)) are black. Other cells, gray. Interactive rendering available: https://stanford.io/2WXR811 d Percent of each BEC subset classified as dividing based on pooled expression of cell-cycle genes. Points represent values for individual samples. Diamonds, average of all samples. Error bars, standard error of the mean. $n=3$ biologically independent samples. e Phenotype of dividing cells presented as percent of dividing cells with the indicated BEC phenotypes. Error bars, standard error of the mean. $n=3$ biologically independent samples. $\mathbf{f}$ Experimental timeline for $(\mathbf{g})$ and $(\mathbf{h})$. $\mathbf{g}$ Quantification of ER ${ }^{+}$endothelium by immunofluorescence histology in resting (day 0) PLN and in PLN 5 days after cutaneous inflammation. Expressed as $\mathrm{ER}^{+}$capillaries (PODXL+ or $\mathrm{MCAM}^{+} \mathrm{PNAd}^{-} \mathrm{EC}^{+}$) or ER ${ }^{+}$ HEV (PNAd ${ }^{+}$) as percent of capillary EC or HEC units (arbitrary units of length) scanned. No ER+ HEC were detected out of over 5000 scanned in resting and over 5000 in inflamed PLN. 95\% CL are shown as error bars or indicated. For each group, nine lymph nodes from two mice were enumerated. Each datum is from a given LN. Statistically significant differences were determined using a Kruskal-Wallis test and Dunn's multiple comparisons test. 95\% confidence limits are shown as error bars or indicated above HEC. $\mathbf{h}$ Representative images of resting PLN from AplnCreER mice stained with anti-PNAd (yellow), anti-PODXL (red), anti-ER (green) and intravenously injected anti-MCAM (blue). Arrow heads point to ER+ CRP. Bars, $50 \mu \mathrm{m}$. Images representative of three independent experiments. i Images of resting PLN ( $72 \mathrm{~h}$ post 4-OHT) from AplnCreER, mTmG mice stained with injected anti-CXCR4 (blue), anti-CD276 (white) and anti-EMCN (red). Arrow heads point to GFP+ cells in capillaries. Bars, $20 \mu$ m. Images representative of three independent experiments.

that CRPs comprise an activated or primed capillary population with the potential to contribute to vascular maintenance and vessel growth. Consistent with this, CRP are enriched in cells with genes for cell division (Fig. $5 \mathrm{~d}$ ). Although only $\sim 10 \%$ of CRP have dividing cell signatures, in resting $\mathrm{LN} \sim 60 \%$ of all cells with gene signatures for cell division align with CRP, with most others distributed to CapEC1 and TrEC (Fig. 5e). Interestingly, division increased as Apln expression declined in the transition from early to late CRP and to TrEC, which lack Apln expression (see below).

As CRP selectively express Apln (Fig. 5a) we localized CRP within the LN vasculature of AplnCreER mice (Fig. 5f) by staining for the human estrogen receptor (ER), which serves as a surrogate of Apln expression in these mice ${ }^{39}$. ER $\mathrm{ER}^{+} \mathrm{EC}$ were readily visualized as thin-walled endothelial cells in capillaries. HEC were not stained by anti-ER, and importantly ER expression detectable by immunofluorescence histology remained restricted to capillary EC after immune challenge (Fig. 5g, Supplementary Fig. 6). ER ${ }^{+}$ CapEC were rapidly labeled by intravenously injected antibodies to surface antigens, consistent with luminal contact and integration into the capillary endothelium (Fig. 5h). CD276 $6^{40}$ and Cxcr $4^{38}$, known markers of angiogenic potential in EC, are also selectively expressed by CRP (Fig. 5a). Anti-CD276 and CXCR4 antibodies injected intravenously labeled a subset of EC limited to capillaries. In AplnCreER x Rosa26 ${ }^{\mathrm{mTmG}}$ (AplnCreER; $\mathrm{mTmG}$ ) mice pulsed three days previously with 4-Hydroxytamoxifen (4-OHT) to induce GFP reporter expression in $\mathrm{AplnER}^{+}$cells, $\mathrm{GFP}^{+} \mathrm{CapEC}$ showed heterogenous staining for injected anti-CD276 and CXCR4 (Fig. 5i).

To assess their fate, we immunized AplnCreER $\times$ R26 $6^{\mathrm{mTmG}}$ mice with Complete Freund's Adjuvant (CFA) 24 h after injection of the short acting tamoxifen metabolite 4-hydroxytamoxifen (4OHT; serum half-life $6 \mathrm{~h}^{41}$ ). Three and a half weeks later, many HEC and capillary EC were positive for the reporter, confirming EC neogenesis from AplnERTCre-expressing precursors (Fig. 6). Similar results were obtained in a repeat experiment in which 4-OHT was administered 3 days prior to immunization (Supplementary Fig. 7) of mice in one leg: GFP expression remained concentrated in scattered CapEC in the control LN even 3 weeks later, whereas numerous $\mathrm{GFP}^{+}$progeny incorporated into $\mathrm{HEV}$ in the immunized node. Finally, consistent with the restricted detection of Apln-promoter-driven ERTCre in CapEC even after immunization (Fig. 5f, g), we found that reporter was selectively induced in capillary EC even when 4OHT was administered $24 \mathrm{~h}$ after immunization: Incorporation of reporter ${ }^{+}$progeny was seen in HEV examined 12 days later (Supplementary Fig. 8). In conjunction with their expression of genes involved in embryonic vascular development and their enrichment in cells undergoing basal cell division, the results suggest that CRP are a poised regenerative subset or progenitor that contributes to vessel maintenance at steady state and to neogenesis of EC including HEC during LN angiogenesis.

We recently identified an Apln-expressing metaphyseal EC subset in the adult bone marrow that contributes to the hematopoietic stem cell niche ${ }^{42}$ : fate mapping in Apln ${ }^{\mathrm{CreER}} \times$ Rosa26 ${ }^{\mathrm{mTmG}}$ mice suggested that the Apln ${ }^{+}$EC contribute to endothelial homeostasis and neogenesis in the marrow vasculature, as shown here for LN CRP. We therefore sought to identify CRP-like EC in BM and other tissues, taking advantage of scRNAseq profiles of BEC from the Tabula Muris (TM) consortium $^{43}$ and the gene expression omnibus (GEO) ${ }^{44,45}$. We performed a global alignment of $\sim 38,000 \mathrm{BEC}$, including cells of our PLN2 and three samples, to cells of our reference PLN1 sample (Supplementary Fig. 9a). We defined candidate CRP-like cells as cells whose gene expression profiles (1) correlated more highly with the mean gene profile of CRP than with other CapEC or differentiated subsets; and (2) aligned with PLN CRP in UMAP projection. A small percentage $(0.3-13 \%)$ of BEC met these criteria in most tissues (Supplementary Fig. 9b): the majority of these expressed Apln as well as other CRP genes including Cd276, Cxcr4, Lxn, Mcam, and Trp53i11 (Supplementary Fig. 9c; Supplementary Data 2). The CRP-like EC express many angiogenic tip-cell-related genes, and like LN CRP share high entropy compared to differentiated BEC in their respective tissues (Supplementary Fig 9c, d). In addition to aligning with LN capillary CRP, most CRP-like cells express capillary-associated gene markers including Gphibp1, Ly6c1, and Cdh13, and lack venous and artery genes. However, BM CRP-like cells had lower $L y 6 c 1$ than cells in other sites. CRP-like EC were extremely rare or absent in liver. In lung, Apln and $P t p 4 a 3$ are highly expressed by capillary aerocytes (also known as alveolar type I capillaries ${ }^{46}$ and Car4-high $\mathrm{EC}^{47}$ ). These cells line alveolar spaces, are unique to the pulmonary vasculature and are otherwise unrelated to CRP. The results suggest that CRP are rare but widely distributed EC of capillary phenotype that likely participate in steady state BEC turnover and function as poised progenitors for induced angiogenesis.

Gene regulation along cellular trajectories. We next examined changes in genes and cell features along cellular trajectories. We isolated cells along paths from early CRP to arteries or to HEC, or from CapEC along the venous branch leading to Vn (Fig. 7a), and visualized expression of genes or gene modules by cells along the trajectories (Fig. 7b-e, Supplementary Fig. 10). The heatmap illustrates enrichment of cells with high cell-cycle scores between 

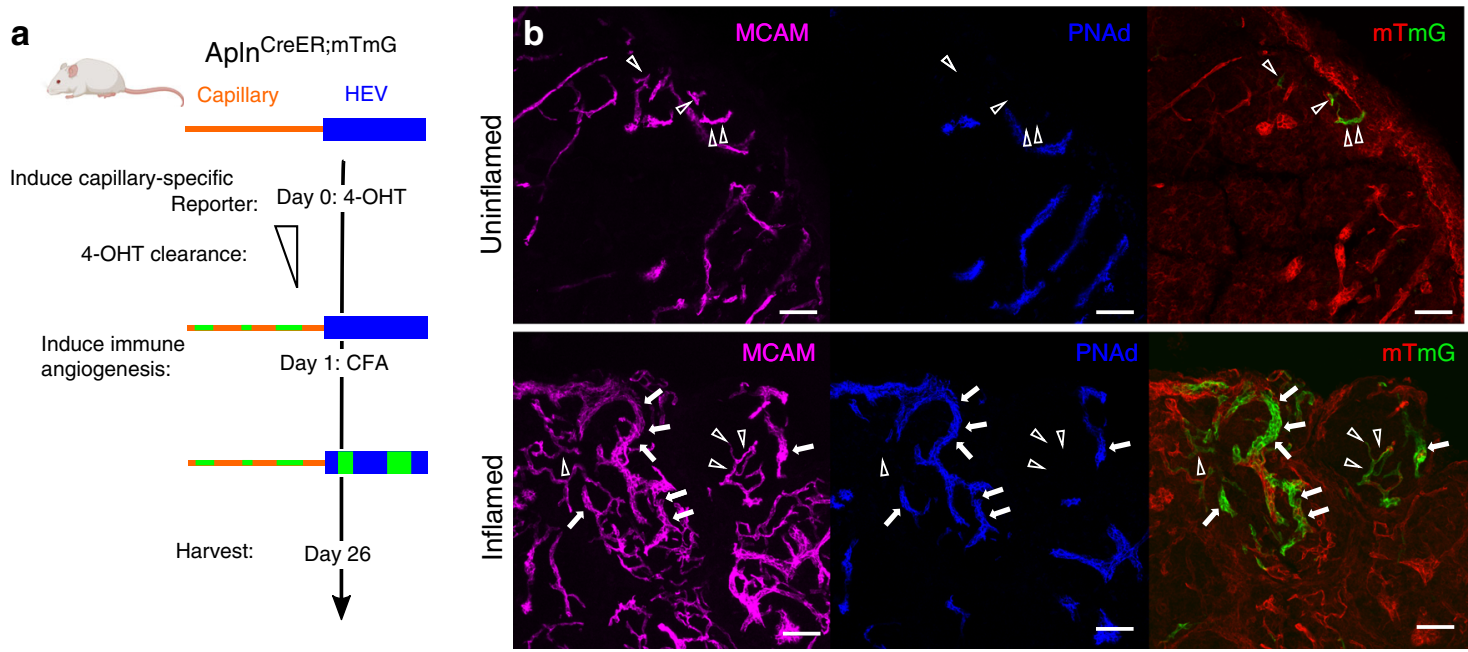

Fig. 6 Lineage tracing of AplnERTCre-expressing capillary EC. a Experimental protocol. b Representative images of Apln-driven reporter expression (GFP) in PLN from Apln-CreER x R26-mTmG mice at rest (upper) and three and half weeks post-immunization with CFA (lower panel). EC subsets were labeled by i.v. injection of the indicated antibodies 10-20 min before sacrifice: PNAd (blue), MCAM (magenta). Arrow heads, capillaries. Arrows, HEV. Bars, $50 \mu \mathrm{m}$.

or along the path from early CRP to CapEC and TrEC, peaking in correspondence with late CRP or the transition to TrEC (Fig. 7b, Supplementary Fig. 10a). Conversely Apln declines rapidly in the transition from early to late CRP and TrEC, and consistent with this dividing CRP have reduced Apln expression compared to early CRP (Fig. 7b, Supplementary Fig. 10a). CapEC on the trajectory to Art express genes implicated in developmental arteriogenesis ${ }^{48}$. These include Sox17, Nrp1, Gata2, Klf2, and genes for Bone Morphogenic Proteins (BMP), Notch and Ephrin signaling components and downstream targets (Acvrl1, Tmem100, Msx1, Notch4 and Notch1, Jag1, Jag2, Hey1, Efnb2) that program the mature arterial phenotype (Fig. 7e). Features reflecting laminar shear stress (Klf2, Klf4, GO:0034616; Fig. 7e) increase along the trajectory to Art, while the oscillatory or gradient shear stress signature peaks in capillary EC, especially the CapEC2-rich region of the trajectory leading to arterial EC (Cxcl1, Egrl, OSS; Fig. 7b, $\mathrm{d}, \mathrm{e})$. Genes involved in gas and metabolite exchange (Aqp ${ }^{49}$, Car2, and Car4) are high in CapEC and lost in the progression to Art. Surprisingly, arterial EC express key genes for elastin fiber assembly (Fbln5, Eln, Ltbp4, and Lox), suggesting that arterial EC may directly participate in the assembly of the inner elastic lamina between EC and smooth muscle cells (Fig. 7d). The CRP to Art trajectory also displays genes involved in arterial EC progenitor migration in development, discussed below.

In contrast, cells along the trajectory from CRP to Vn or HEC express determinants of venous fate and phenotypes. Nr2f2 encodes CoupTF2, a TF required for venous differentiation in development: Nr2f2 is first expressed in TrEC and is maintained along the venous trajectory. Notch is inhibited during venous differentiation ${ }^{13}$, and Notch signaling components are downregulated early along the venous trajectory (Fig. 7d). The lymphotoxin receptor LTBR is required for HEV development and maintenance. Lbtr is broadly expressed, but lymphotoxin beta $(L t b)$, a component of HEV-specifying lymphotoxin dimers, appears in the late HEC portion of the venous axis, suggesting the potential for autocrine signaling to maintain the HEC phenotype (Fig. 7b). In addition to mapping known mediators and mechanisms of arterial and venular specification to cellular trajectories, the analysis identifies candidate transcription factor genes that may contribute to specialization of large vessels (e.g., Ebf1, Klf9), arteries (e.g., Tsc22d1), HEC (e.g., Xbp1, Meox1), HEC and vein (Aebp1) or veins (Lhx6, Csrnp1, Gata6) (Fig. 7d). For example, Gata6 enhances Tumor Necrosis Factor-alpha induced VCAM1 expression in EC cultures ${ }^{50}$ and its expression by TrEC and $\mathrm{Vn}$ may thus contribute to selective Vn expression of Vcam1.

G-protein-linked receptors (GPCR) serve as environmental sensors. With their ligands GPCRs regulate vascular development, endothelial function, and migration. We examined expression of GPCR along the aligned trajectories. The vasodilatory flow sensor gene Gpr68 ${ }^{51}$ first arises in pre-Art capillary EC and is retained in Art (Fig. 7b). Cysltr1, encoding a sensor for myeloid cell-derived leukotrienes ${ }^{52}$, is upregulated in the transition to $\mathrm{Vn}$ (Fig. 7b). Genes encoding thrombin and erythropoietin receptors ( $F 2 r$ and Epor), sensors that drive angiogenesis and vasculogenesis ${ }^{53,54}$, are expressed selectively by CRP, suggesting sensitivity to local thrombotic or inflammatory protease activity and oxygen deficiency (Fig. 7b). Patterns of expression of genes for Cxcl12 (in CapEC and pre-Art), and for its chemoattract receptor Cxcr4 (expressed by CRP and subsets of CapEC1 and 2 along the trajectory to Art) suggest retention of a developmental programs for tip-cell migration along capillaries into developing arteries (Fig. 7c), as observed during retinal arteriogenesis ${ }^{55}$. Ackr3 expression by trailing cells is required for Cxcr4/Cxcl12-dependent migration of germ cells in zebrafish development ${ }^{56}$. Ackr3 is expressed by CRP and some CapEC in the arterial branch, suggesting a parallel role. These observations suggest that CRP may be primed for migration. Consistent with this, CRP are enriched in genes for cell locomotion, extracellular matrix remodeling, actin assembly and disassembly and lamellipodium formation (Fig. 7e); but they also express genes for known inhibitors of migration and sprouting behavior including Arhgap $18^{57}$ and Csnk2b $b^{58}$ (Supplementary Data 1).

\section{Discussion}

The vascular endothelium plays a central role in lymphoid tissue development and function. Our survey of the LN blood vascular endothelium defines the diversity of EC phenotypes and identifies previously undescribed cell subsets and functions. We uncover multiple subsets of capillary endothelial cells with distinct gene expression including TrEC, a transitional phenotype subset intermediate in gene expression and in physical location between 
a
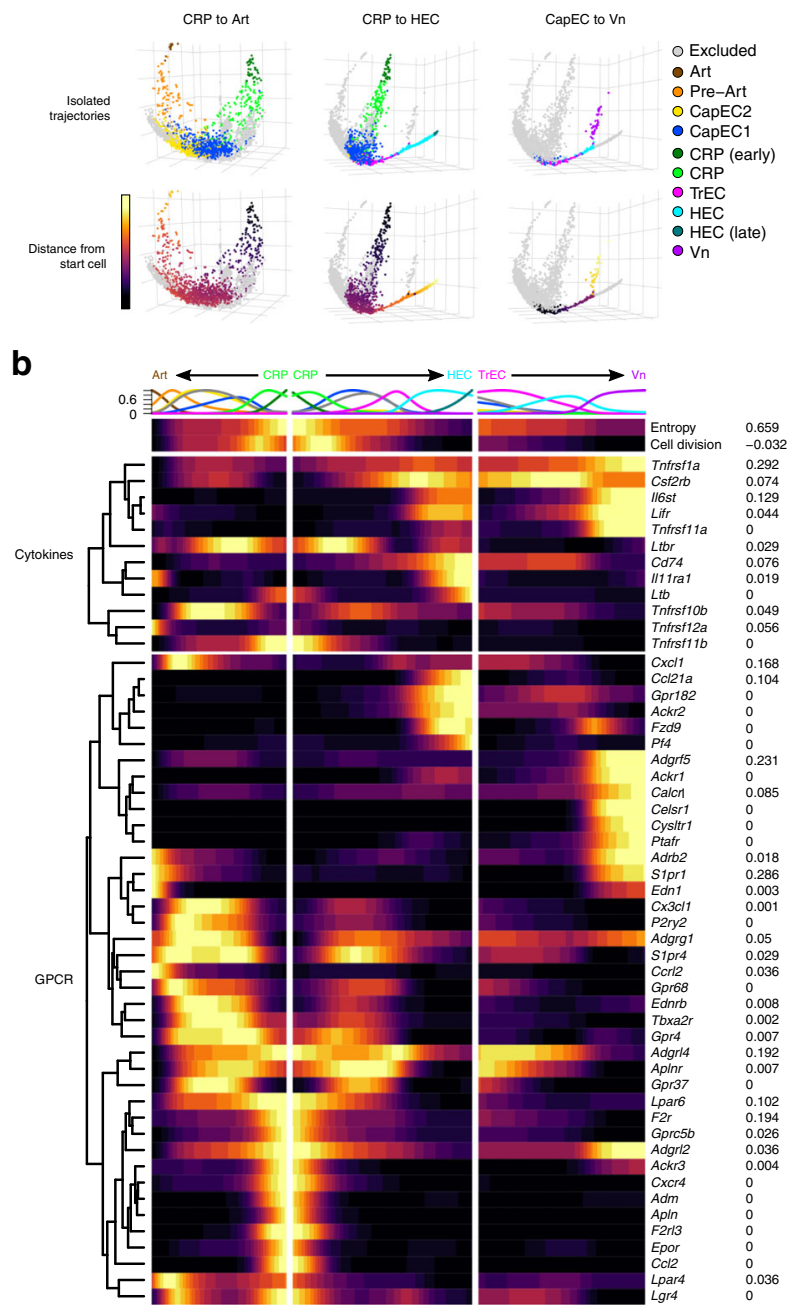

e

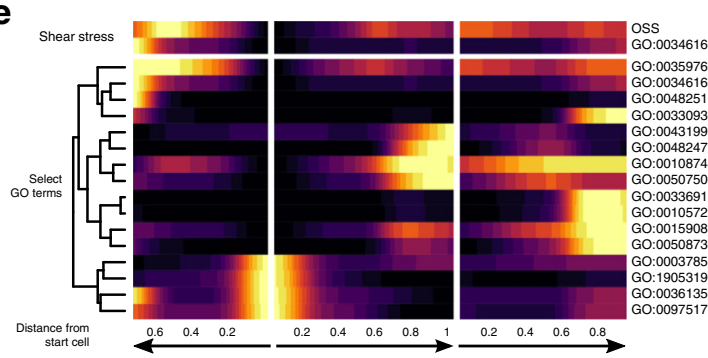

C

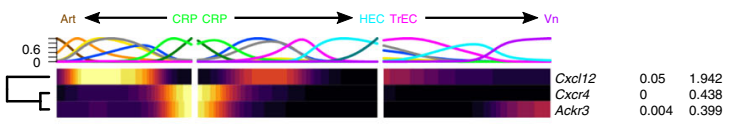

d

deGF
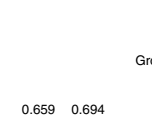

Growin factors
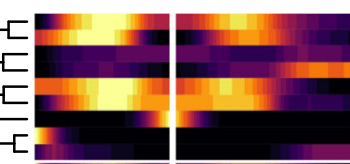

ᄃ

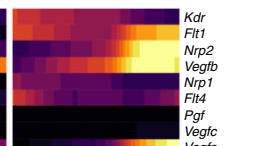

0.21
0.197
0.1 .999

$\begin{array}{lll}0.197 & 1.194 \\ 0 & 0.455 \\ 0.004 & 0.064\end{array}$

$\begin{array}{ll}0.004 & 0.064 \\ 0.061 & 0.759 \\ 0 & 0.035\end{array}$

0.035
0.293
0.551
0.086

$\begin{array}{lll}0.086 \\ 0.162 & 0.349 \\ 0.71 & 0.43\end{array}$

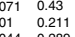

$\begin{array}{ll}.044 & 0.289 \\ 0.009 & 0.065 \\ 0.072 & 0.405 \\ 0.405\end{array}$

$\begin{array}{ll}0.072 & 0.405 \\ 0.014 & 0.349 \\ 001 & 0.163\end{array}$

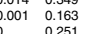

\section{$\begin{array}{ll}0.292 & 0.737 \\ 0.074 & 0.455\end{array}$}

$\begin{array}{lll}0.074 & 0.455 \\ 0.129 & 1.412\end{array}$

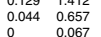

$\begin{array}{ll}0.029 & 0.067 \\ 0.076 & 0.128 \\ 0.019 & 0.183\end{array}$

$\begin{array}{ll}0.019 & 0.13 \\ 0 & 0.211 \\ 0.049 & 0.201 \\ 0.056 & 0.143\end{array}$

$\begin{array}{ll}0 & 0.09 \\ 0.168 & 0.93 \\ 0.104 & 4.989\end{array}$

$\begin{array}{lll}0.104 & 4.989 \\ 0 & 0.37 \\ 0 & 0.051 \\ 0 & 0.016\end{array}$

$\begin{array}{ll}0 & 0.051 \\ 0 & 0.016 \\ 0 & 0.741 \\ 0.231 & 0.749 \\ 0 & 0.529 \\ 0.085 & 0.571 \\ 0 & 0.068 \\ 0 & 0.028\end{array}$

$\begin{array}{ll}0.085 & 0.571 \\ 0 & 0.068 \\ 0 & 0.231 \\ 0 & 0.079 \\ 0.018 & 0.35 \\ 0.286 & 0.881 \\ 0.280 & 0.896\end{array}$

$\begin{array}{ll}0.003 & 0.696 \\ 0.001 & 0.273 \\ 0 & 0.091 \\ 0.05 & 0.239\end{array}$

$\begin{array}{ll}0 & 0.091 \\ 0.05 & 0.239 \\ 0.029 & 0.08 \\ 0.036 & 0.179\end{array}$

$\begin{array}{ll}0.036 & 0.179 \\ 0 & 0.042 \\ 0.008 & 0.413 \\ 0.002 & 0.068\end{array}$

$\begin{array}{ll}0.002 & 0.068 \\ 0.007 & 0.113 \\ 0.192 & 0.536\end{array}$

$\begin{array}{lll}0.007 & 0.113 \\ 0.192 & .036 \\ 0.007 & 0.631 \\ 0 & 0.02 & 0.45\end{array}$

$\begin{array}{ll}0 & 0.046 \\ 0.102 & 0.499 \\ 0.194 & 0.77 \\ 0.026 & 0.182\end{array}$

$\begin{array}{ll}0.036 & 0.28 \\ 0.004 & 0.399 \\ 0 & 0.438\end{array}$

$\begin{array}{ll}0 & 0.438 \\ 0 & 0.223 \\ 0 & 0.405 \\ 0 & 0.059 \\ 0 & 0.059 \\ 0 & 0.039 \\ 0 & 0.054 \\ 0.036 & 0.154 \\ 0 & 0.039\end{array}$

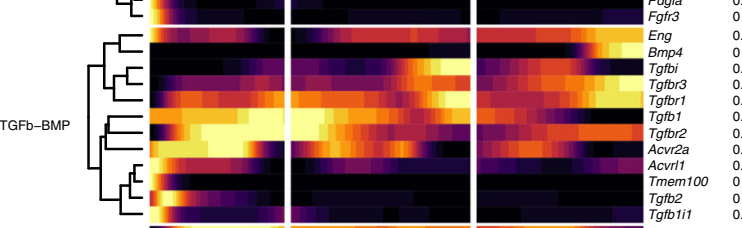

$\begin{array}{ll} & 0.251 \\ 033 & 1.272 \\ 0.167 \\ 0\end{array}$

$\begin{array}{lll}0.021 & 0.167 \\ 0.0195 \\ 0.04 & 0.235 \\ 0.175 & 0394\end{array}$

$\begin{array}{lll}.0 .044 & 0.219 \\ 0.175 & 0.384 \\ 0.384 & \end{array}$

$\begin{array}{lll}0.175 & 0.384 \\ 0.006 & 0.271 \\ .008 & 0.058 \\ 0 & 0.075\end{array}$

$\begin{array}{lll}0.143 & 0.634 \\ 0 & 0.775 \\ 0 & 0.255 \\ 0.972 & 0.52\end{array}$
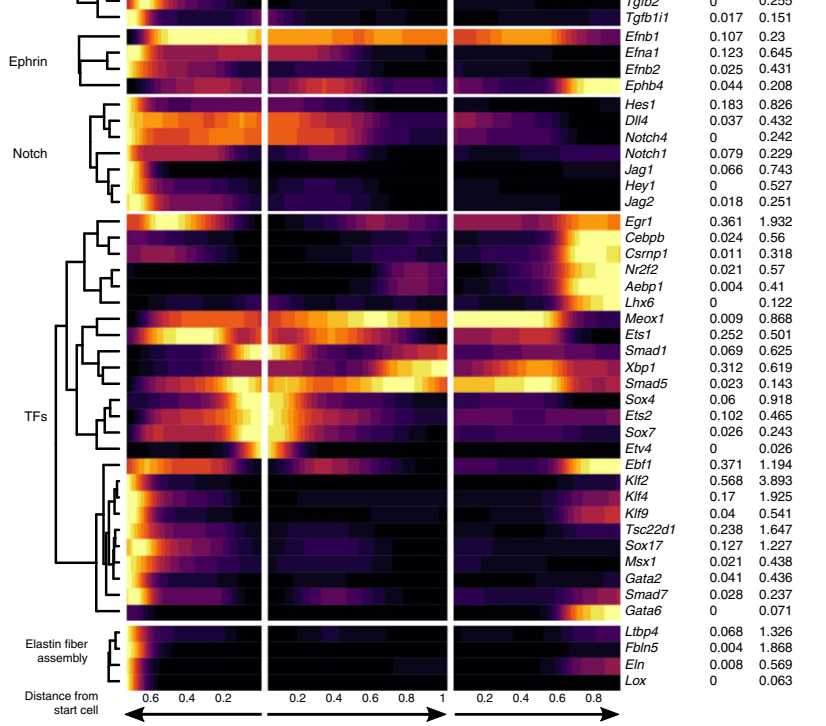

$\begin{array}{ll}0.066 & 0.743 \\ & 0.527 \\ 0 & 0.251\end{array}$

$\begin{array}{ll}0.018 & 0.251 \\ 0.361 & 1.932 \\ 0.024 & 0.56\end{array}$

$\begin{array}{ll}0.361 & 1.932 \\ 0.024 & 0.56 \\ 0.011 & 0.318\end{array}$

$\begin{array}{ll}0.011 & 0.318 \\ 0.021 & 0.57\end{array}$

$\begin{array}{ll}0.021 & 0.57 \\ 0 & 0.122 \\ 0.009 & 0.868 \\ 0.025 & 0.51\end{array}$

$\begin{array}{ll}0.009 & 0.868 \\ 0.252 & 0.501\end{array}$

$\begin{array}{ll}0.069 & 0.625 \\ 0.312 & 0.619 \\ 0.023 & 0.143\end{array}$

$\begin{array}{ll}0.023 & 0.143 \\ 0.06 & 0.918\end{array}$

$\begin{array}{ll}0.102 & 0.465 \\ 0.026 & 0.243\end{array}$

$\begin{array}{ll}0 & 0.026 \\ 0.371 & 1.194 \\ 0.17 & 1.925\end{array}$

$\begin{array}{ll}0.568 & 3.893 \\ 0.17 & 1.925\end{array}$

$\begin{array}{ll}0.04 & 0.541 \\ 0.238 & 1.647 \\ 0.127 & 1.227 \\ 0 & 0.047\end{array}$

$\begin{array}{ll}0.021 & 0.438 \\ 0.041 & 0.436 \\ 0.028 & 0.37\end{array}$ $\begin{array}{ll}0.028 & 0.237 \\ 0 & 0.071\end{array}$ $\begin{array}{ll}0.068 & 1.326 \\ 0.004 & 1.868\end{array}$ $\begin{array}{ll}0.004 & 1.868 \\ 0.008 & 0.569 \\ 0 & 0.063\end{array}$

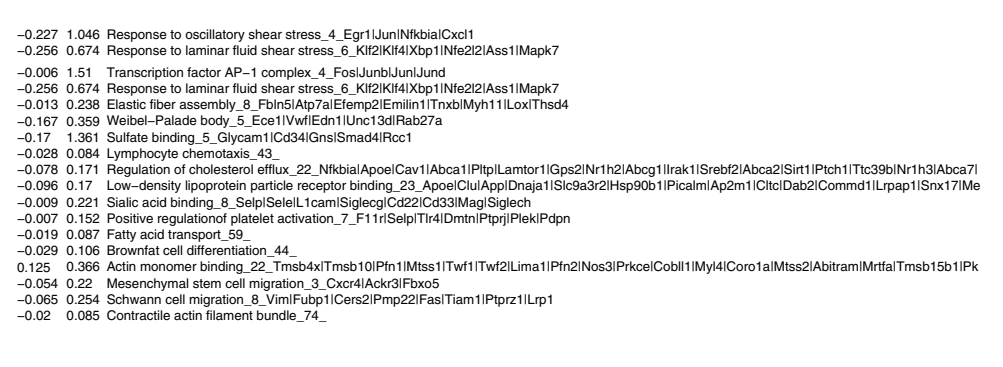

-0.2271 .046 Response to oscillatory shear stress_4_EgrilununINfkbialCxcl1

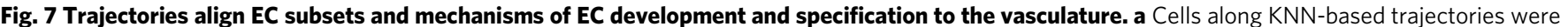
isolated (Methods). b-e Expression of selected genes and gene set enrichment scores along cell trajectories from mature CRP to Art (plotted leftward), and from CRP to HEC or Vn (rightward). Cells along the trajectories were manually gated in the first five principal components of trajectory space and aligned according to distance from early CRP. Representation of cell types along the trajectories is indicated at top. Normalized count data plotted as a function of trajectory distance was smoothed using a gaussian kernel. Trajectory distances were scaled to the longest trajectory (CRP to HEC). Genes were grouped according to biological class or function. Imputed gene expression values for all cells (without duplications) were calculated independently and used for hierarchical clustering within each gene group. Cxcr4, Cxcl12, and Ackr3 are shown as a separate group at the top in c (see results). Average normalized expression values for the min and max subset are indicated to the right of each heatmap. OSS: pooled expression of oscillatory shear stress genes: Egr1, Nfkbia, Junb, Cxcl1. Cell Division: pooled expression of cell-cycle genes ${ }^{74}$.

other CapEC and HEC. We show that this subset expresses glycotopes $^{15}$ for lymphocyte tethering under flow. Presentation of tethering glycotopes by capillary EC immediately upstream of HEC may allow lymphocytes to initiate interactions with the endothelium prior to entering HEV. We identify a unique profile of medullary venous EC and show that they explicitly recruit myeloid cells and not lymphocytes to the LN medulla in response to acute bacterial challenge. Neutrophils block pathogen spread beyond the initial tissue draining $\mathrm{LN}^{18}$, and neutrophils recruited into the medulla may be well positioned to intercept bacteria prior to their exit into the efferent lymphatics. Finally, we identify a primed capillary resident population, CRP, that display features 
associated with multipotent progenitor cells and participate in basal endothelial proliferation and in vascular neogenesis in response to immunization.

New endothelium in physiologic angiogenesis is thought to arise principally from local $\mathrm{EC}^{59}$, and circulating endothelial progenitors do not contribute to new endothelium in immunized $\mathrm{LN}^{4}$. Prior studies have highlighted multipotent vessel-resident EC progenitor populations in large vessels ${ }^{60,61}$ and lung ${ }^{62}$ and precursors in developing bone ${ }^{63}$ that can differentiate into diverse EC phenotypes. CRP appear distinct from these populations: They lack Bst1 and Procr, markers of resident endothelial progenitors described in large vessels of liver ${ }^{60}$ and fat ${ }^{61}$. They express Kit, a marker of clonally proliferative EC progenitors in lung ${ }^{64}$; but published single-cell datasets of lung blood vascular EC include only extremely rare EC with CRP-like gene profiles. CRP are also distinct from angiogenic tip cells in that CRP are in direct contact with the lumen and are integral to the vessel lining, whereas tip cells lack luminal contact, instead leading the blind end of invasive sprouts ${ }^{65}$. However, CRP share gene expression and precursor potential with tip cells, and thus may be primed for tip-cell behavior or alternatively for intussusceptive (splitting) angiogenesis. CRP appear closely related to Apln-expressing EC we have observed in the adult bone marrow, which fate mapping suggests contribute to irradiation-induced neogenesis of EC including arterial EC in the marrow compartment ${ }^{42}$. Moreover, we identify rare CRP-like capillary phenotype EC in many tissues. Thus CRP may contribute widely to vascular homeostasis, providing a distributed pool of regenerative cells for local vascular maintenance and replenishment. Consistent with this thesis, a recent scRNAseq study also noted rare Apln+ "angiogenic" EC in normal tissues ${ }^{66}$.

Interestingly, in PLN and in most tissues profiled Apln expression appears quite selective for CRP or CRP-like EC. At the gene level, its expression is highest in 'early' CRP, which also show the highest entropy among the BEC profiled here. Apln is downregulated progressively in cells along the trajectory from early to late CRP and CapEC; and its translation to protein as assessed by Apln-promoter-driven expression of CreERT2 remains restricted to capillaries even in immunized lymph nodes, in which many HEV and other BEC are undergoing active proliferation. Thus Apelin is not a general marker of EC activation or proliferation. These considerations emphasize the similarities between early CRP and tip cells in models of sprouting angiogenesis: tip cells like early CRP are Apln high, predominantly non-dividing and display signatures of cell migration; whereas cell division occurs primarily among trailing 'stalk' cells 67 .

The migratory signature of CRP suggests that they may have the capacity to crawl within capillaries to contribute to new vessel formation. We show that CRP express genes for locomotion including a Cxcr4 chemoattractant program with the potential to drive precursor migration toward pre-artery-expressed $\mathrm{Cxcl} 12$ for arteriogenesis, as observed for tip-cell progeny in retinal development ${ }^{68}$. We also show CRP expression of Ackr3 encoding the Cxcl12 interceptor Ackr3 (CXCR7): CXCR7 internalizes CXCL12, and in developing cell systems can enhance CXCR4driven directional migration by reducing chemoattractant levels at the "rear" of a migrating population 69 .

CRP display sensory receptors for angiogenesis signals, including thrombin, erythropoietin and VEGFs, and thus appear well programmed to respond to requirements for endothelial proliferation. Consistent with this, we show that nearly half of all BEC with basal cell division signatures align with CRP, predominantly late CRP as just mentioned. CRP may represent a temporary state of activated capillary EC, as suggested by enrichment in dividing cells and similarities to tip cells in their gene expression. Alternatively, they may represent a resident progenitor pool, analogous to regenerative stem or progenitor cells in other settings (i.e., intestinal epithelium and hematopoietic systems). They embody high entropy and express genes characteristic of multipotent progenitors and stem cells. Serial cell transfer or clonal culture will be required to evaluate their clonal progenitor potential and capacity for self-renewal. Our data do not address the precursor potential, in terms of contribution to new vessel formation, of the other EC subsets defined here. CapEC1, TrEC, and early HEC display higher signaling entropy than the most differentiated arterial and venous EC in our samples, suggesting that they may also retain developmental plasticity. Indeed, HEC have been reported to contribute to neosynthesis of HEC and capillary cells when injected into a recipient mouse $\mathrm{LN}^{4}$. In the intestinal epithelium recently differentiated cells can even re-acquire multipotency and renew stem cell pools in response to injury or stem cell depletion ${ }^{70}$ : Some subsets of BEC may be capable of doing so as well. The relative contributions of different $\mathrm{BEC}$ subsets to neosynthesis of specialized EC may be a function of the tissues they reside in and the challenges they experience.

We also observed differences among the major CapEC pool, defining two related clusters, CapEC1 and CapEC2. Both share canonical CapEC genes and genes for gas and metabolite transport, which are downregulated in pre-artery and terminal arterial subsets. CapEC1 feature high expression of inhibitor of DNA binding proteins (Id1, Id3), proteins that act as inhibitors of cell differentiation. CapEC2 express genes (e.g., Egr1, Cxcl1) and pathways (NFkB, JNK, WNT, MAPK cascade) induced by oscillatory or gradient shear stress, which may occur at vessel bifurcations. Interestingly, these genes decline along a trajectory from CapEC2 to arterial EC, while laminar flow associated genes Klf2 and Klf4 progressively increase. Pre-Art may thus correspond to the arterioles and potentially to cells lining arteriovenous communications in the LN.

The ability of medullary veins to selectively recruit myeloid cells, and the selective expression and role of vascular selectins in the process, reveal strikingly local vascular specialization. Previous studies have shown that inflammatory stimuli induce de novo monocyte and neutrophil recruitment to $\mathrm{LN}$ but have focused on the role of HEV and the HEV L-selectin ligand PNAd which recruit cells preferentially into the deep cortex ( $T$ cell zones). We showed recently for example that neutrophils home via $\mathrm{HEV}$ into $S$. aureus challenged $\mathrm{LN}$ in a PNAd-dependent, vascular selectin-independent process ${ }^{18}$. In contrast, we find here that medullary veins lack the machinery for naïve lymphocyte homing, instead selectively recruiting myeloid cells using the vascular selectins. The medulla of the lymph node contains pathogen-trapping lymphatic EC networks that we have recently characterized at the single-cell level ${ }^{71}$ : recruitment of neutrophils to the medulla in acute bacterial challenge may contribute to the essential role of myeloid cells in reducing pathogen transit into efferent lymphatics and systemic spread of infection.

Our results show that the major EC subsets, defined by gene signatures, map to specific locations within the steady state vasculature. Indeed, alignment of cells along nearest neighbor trajectories recapitulates the overall architectural arrangement of EC in the blood vasculature. Imaging confirms the computationally predicted positioning of TrEC between CapEC and HEV; of CRP within capillary segments; and the branching of PNAd negative veins from HEV. Trajectory analysis also reveals that capillary EC aligned along trajectories to mature arterial EC express transcriptional programs that, in development, support arteriogenesis. Similarly, genes that program developmental specification of veins are retained along the venous branch. This 'retention' of artery and venous specifying programs may reflect the continuous 
steady state replenishment and developmental programming of these specialized subsets from dividing CRP or other precursors. Alternatively, retention of developmental genes could serve to pre-program segmental EC differentiation for rapid vascular expansion during immune angiogenesis.

As shown here, single-cell analysis has the potential to identify EC subsets; elucidate developmental processes, transcriptional and regulatory pathways that program their specialization; and map transcriptional phenotypes to the vasculature, providing a molecular blueprint of the vascular endothelium. Targeting specific subsets and processes defined here holds promise to treat a variety of vascular, immune and inflammatory disorders through manipulation of angiogenesis and immune responses.

\section{Methods}

Mice. Apln $\mathrm{CreER}$ (apelin; targeted mutation 1.1) ${ }^{39}$, BALB/cJ (The Jackson Laboratory), B6.Cg-Gt(ROSA)26Sortm9(CAG-tdTomato)Hze/J (The Jackson Laboratory), and B6.129(Cg)-Gt (ROSA)26Sortm4(ACTB-tdTomato,-EGFP)Luo/J (The Jackson Laboratory) mice were bred and maintained in the animal facilities at Veterans Affairs Palo Alto Health Care System, accredited by the Association for Assessment and Accreditation of Laboratory Animal Care. Experimental protocols were approved by the Stanford University Institutional Animal Care and Use Committee. Male and female mice between the ages of 8-20 weeks were used in experiments. LysM ${ }^{\mathrm{GFP}}$ mice (lysozyme 2 ; targeted mutation 1.1 ; kindly provided by Dr. Thomas Graf) were maintained in a specific pathogen-free environment at the University of Calgary Animal Resource Centre.

Preparation of lymphoid tissue BECs for flow cytometry. Axillary, inguinal and brachial PLN from 20-30 adult mice: BALB/cJ (PLN1 and PLN2) or mice of mixed background (PLN3) were dissociated as described ${ }^{6}$. To minimize technical variation, in one study (PLN1) male and female PLN were combined before processing the tissue and separated postsequencing using the AddModuleScore function from the Seurat package (v3.1.1) to calculated enrichment of male-specific genes (y-chromosomal genes) and the female-specific gene Xist. Endothelial cells were isolated essentially as described (online version) ${ }^{6}$. In brief, PLN were pooled in HBSS buffer and minced with scissors. Minced tissue was washed 2-3 times with HBSS, resuspended in HBSS media containing $0.2 \mathrm{mg} / \mathrm{ml}$ collagenase $P, 0.8 \mathrm{mg} / \mathrm{ml}$ Dispase II, $0.01 \mathrm{mg} / \mathrm{ml}$ DNase, and incubated at $37^{\circ} \mathrm{C}$ for $10 \mathrm{~min}$. Tissue was then gently disrupted by pipetting up and down several times through P1000 pipette tips with successively smaller bore sizes. Tissue was allowed to settle to the bottom of the tube and cells released by the digestion were transferred to ice cold FBS (final concentration $30 \%$ ). Fresh digestion buffer was added to the remaining tissue fragments for an additional round of digestion. Digested cells were filtered and hematolymphoid cells were depleted from the cell suspension using anti-CD45 MicroBeads (Miltenyi). Approximately, 5-10 $\times 10^{4} \mathrm{BECs}\left(\operatorname{lin}^{-} \mathrm{Gp} 38^{-} \mathrm{CD} 31^{+}\right.$) were sorted into $100 \%$ fetal bovine serum using a FACS Aria (100 $\mu \mathrm{m}$ nozzle; 2500 cells/second). Freshly sorted cell suspensions were diluted with PBS to a final FBS concentration of $\sim 10 \%$ and centrifuged at $400 \times g$ for $5 \mathrm{~min}$. Supernatant was carefully removed using micropipettes and cell pellets resuspended in the residual volume $(\sim 30-50 \mu \mathrm{l})$. Cells were counted using a hemocytometer and cell concentration adjusted to $500-1000$ cells $\mu$ l) by addition of PBS with 10\% FBS if necessary.

Single-cell RNA sequencing. Cell suspensions were processed for single-cell RNA sequencing using Chromium Single Cell 3' Library and Gel Bead Kit v2 (10X Genomics, PN-120237) according to 10X Genomics guidelines. Libraries were sequenced on an Illumnia NextSeq 500 using 150 cycles high output V2 kit (Read 1-26, Read2-98 and Index 1-8 bases). The Cell Ranger package (v3.0.2) was used to align high quality reads to the $\mathrm{mm} 10$ transcriptome (quality control reports available: https://stanford.io/37sXZV3). Normalized log expression values were calculated using the scran package ${ }^{72}$. Imputed expression values were calculated using a customized implementation (https://github.com/kbrulois/magicBatch) of the MAGIC (Markov Affinity-based Graph Imputation of Cells) algorithm ${ }^{73}$ and optimized parameters $(\mathrm{t}=2, \mathrm{k}=9, \mathrm{ka}=3)$.

Highly variable genes were identified using the FindVariableGenes function (Seurat, v2.1 $)^{27}$. For analyses designed to identify clusters, non-variable genes, cellcycle genes ${ }^{74}$, genes detected in fewer than three cells and genes with an average expression level below 0.3 (normalized and log-transformed counts) were excluded. Supervised cell selection was used to remove cells with non-blood endothelial cell gene signatures: lymphatic endothelial cells (Prox1, Lyve1, Pdpn); Pericytes (Itga7, Pdgfrb); fibroblastic reticular cells (Pdpn, Ccl19, Pdgfra); lymphocytes (Ptprc, Cd52). Top principal components and the FindClusters function (Seurat, v2.1; res $=0.3$ ) were used on a core set of cells (2394) from the PLN1 sample to identify the 8 major clusters. The Arterial, HEC and CRP clusters were further subdivided into Art and Pre-Art, HEC and HEC (late); and CRP and CRP (early), based on canonical marker expression and their position in tSpace projections of PLN1, yielding a total of 11 subsets. The remaining PLN1 cells and cells from the independently processed samples (PLN2 and PLN3) were assigned the identity of the maximally correlated (Pearson) average expression profile of the core PLN1 cell subsets using $\sim 3000$ common variable genes. Batch effects from technical replicates were removed using the $\mathrm{MNN}$ algorithm ${ }^{75}$ as implemented in the batchelor package's (v1.0.1) fastMNN function. Cells were classified as dividing or resting using a pooled expression value for cell-cycle genes (Satija Lab Website:

regev_lab_cell_cycle_genes). For UMAP and tSpace embeddings, cell-cycle effects were removed by splitting the data into dividing and resting cells and using the fastMNN function to align the dividing cells with their resting counterparts. Dimensionality reduction was performed using the UMAP algorithm and nearest neighbor alignments for trajectory inference and vascular modeling were calculated using the tSpace algorithm ${ }^{10}$. Cells along isolated trajectories were selected by gating within tPC projections 1-5; and illustrated in the figures here. Differential gene expression analysis was performed by comparing each subset to the remaining cells and fitting a zero-inflated negative binomial model using the LineagePulse package, v0.99.20. In order to assess differentiation potency of single cells, the signaling entropy rate (SR) of each cell was calculated ${ }^{36}$ after random downsampling of reads to 1000 reads/cell.

Data visualization. Heatmaps were generated using the ComplexHeatmap package $^{76}$, scaled to a maximum value of 1 . Data for trajectory heatmaps was preprocessed using code adapted from the plot_as_function from cyt (Pe'er Lab): normalized count data was smoothed with respect to trajectory distance using a gaussian kernel and plotted using a discrete color scale. Violin plots were generated using ggplot2; $y$-axis units for gene expression data correspond to logtransformed normalized counts after imputation. $3 \mathrm{~d}$ plots were generated using the rgl package v0.100.30, with minor source code modifications for interactive renderings.

GO term analysis. Pooled expression values for GO term gene sets and other sets of genes were calculated as previously described ${ }^{77}$ using the AddModuleScore function of Seurat (v3.1.1), which centers values by subtracting pooled expression for random sets of control genes with similar expression levels. To identify biologically relevant GO terms, we first generated a pooled expression matrix by systematically applying the AddModuleScore function to all currently annotated GO terms with at least 3 expressed genes. Differentially regulated GO terms were identified from the resulting "GO term expression matrix" (14300 GO terms by 8832 cells) by comparing each subset to the remaining cells using a Student's $t$-test. A high degree of overlap was observed with conventional GO term analysis approaches such as analysis of top differentially expressed genes using Enrichr. Because this GO term analysis approach is done on a cell by cell basis, it was particularly useful for the identification of terms whose enrichment spanned multiple subsets, e.g., GO:0015669_gas transport (Supplementary Fig. 3).

Antibodies. The following antibodies (clone; catalogue number; dilution) were used for both microscopy and FACS: Brilliant Violet (BV) 605-conjugated CD31 (390; 102427; 1:100), peridinin chlorophyll protein-cyanine 5.5-conjugated antiCD45 (30-F11; 103131; 1:100), peridinin chlorophyll protein-cyanine 5.5-conjugated anti-Ter-119 (TER-119 TER-119; 116227; 1:100), peridinin chlorophyll protein-cyanine 5.5-conjugated anti-CD11a (H155-78; 141007; 1:100), peridinin chlorophyll protein-cyanine 5.5-conjugated anti-CD326 (G8.8; 118219; 1:100), phycoerythrin-cyanine 7-conjugated anti-Gp38 (8.1.1; 127411; 1:100), antiVE-Cadherin (VECD1; 138101), and BV421-conjugated anti-CXCR4 (L276F12; 146511) were from Biolegend. BV421-conjugated anti-CD146 (ME-9F1; 740095; 1:100) and BV480 Streptavidin (564876; 1:200) were from BD Biosciences. Antiestrogen receptor alpha antibody (SP1; ab16660; 1:100) and Anti-ERG antibody (EPR3864; ab196149 and ab196374) were from Abcam. Anti-CD276 (MIH35; 165937-81) was from Thermo Fisher Scientific. Anti-PNAd (MECA79), anti-Ly6c (Monts1), anti-EMCN (5C7), anti-PODXL (MECA-99), anti-ICAM-1 (BE29G1), anti-VCAM-1 (6C2.1), anti-PLVAP (MECA-32), and anti-Slex (F2) were produced in-house from hybridomas; labelled with DyLight Antibody Labeling Kits or Biotin labeling kit (Thermo Fisher Scientific), and used at a concentration of $10 \mathrm{ug} / \mathrm{ml}$. Alexa Fluor 488-conjugated donkey antibody to rabbit IgG (711-546-152; 1:500) was from Jackson ImmunoResearch Laboratories. Antibodies for in vivo blockade of selectins were from BD Biosciences: Anti-E-selectin (10E9.6; 553749), anti-Pselectin (RB40.34; 550289), and NA/LE isotype control rat IgG1 (R3-34; 554682). Antibody dilutions for FACS are approximate; actual dilution varied between experiments.

Imaging. PLN were imaged following either retroorbital injection of fluorescent labeled antibodies or by fluorescence staining of LN sections. If injected, antibodies $(25-75 \mu \mathrm{g})$ were administered 5-30 min prior to sacrifice and PLN removal. To image the overall vasculature, the PLN was gently compressed to $\sim 35-50 \mu \mathrm{m}$ thickness on a glass slide. Alternatively, PLNs were fixed with $4 \%$ paraformaldehyde, cryoprotected with sucrose, frozen in OCT (Sakura ${ }^{\circledR}$ Finetek) in 2methylbutane (Sigma) on dry ice and stored at $-20^{\circ} \mathrm{C} .50 \mu \mathrm{m}$ cryo-sections were stained with antibodies according to standard protocols. The slides were imaged 
using Apotome 2.0 fluorescence microscope or LSM 700 or LSM 880 laser scanning microscope (Zeiss). Noise was removed using Fiji.

For quantification of $\mathrm{ER}^{+}$cells, anti-human estrogen receptor antibody was used as a surrogate stain for Apln in sections from AplnCreER mice. Capillaries, $\mathrm{HEV}$ and $\mathrm{ER}^{+}$vessels were enumerated within $50 \mu \mathrm{m}$ sections at $\times 20$ objective using a grid reticle to determine the relative frequency of each EC subset (1 length unit $=1 / 8$ of the grid height). Sufficient fields were scanned to comprise $>5000$ HEC assessed for reactivity with anti-ER antibody. Data were expressed as frequency of $\mathrm{ER}^{+}$(as a \% of total counted vessels, i.e., length of $\mathrm{ER}^{+} /$length of capillaries + HEC) per lymph node section. We scanned nine LN from two Apln $\mathrm{CreER}$ unchallenged mice and nine $\mathrm{LN}$ from two AplnCreER mice five days post cutaneous inflammation (one section per LN). The number of HEC per field was determined based on $\mathrm{ERG}^{+}$nuclei within MECA79 ${ }^{+}$EC using $\times 10$ or $\times 20$ objectives.

Live imaging. LysM $\mathrm{MFP}^{\mathrm{GF}}$ mice were injected in the right footpad with $2.5 \times 10^{7} \mathrm{CFU}$ S. aureus. An hour later mice received intravenously injected Cell Tracker Red CMTPX (Thermofisher) labeled lymphocytes. An hour after lymphocyte injection mice were injected intravenously with a mixture of anti-PNAd ${ }^{-}$Dylight594 and albumin-Dylight-680 to visualize HEV and vasculature, respectively. Two-photon videomicroscopy was employed to assess the movement and location of neutrophils and lymphocytes in lymph node blood vessels following infection with S. aureus. The right hindlimb popliteal LN was exposed for imaging in the anesthetized mouse. The LN was imaged from 2 to 4 hpi. Image acquisition was performed an upright two-photon microscope (Leica Biosystems TCS SP8 Upright Microscope). For antibody blocking studies, mice were pretreated with blocking antibody 20 min before infection.

Lymph node immunization. Oxazolone: Mice were subjected to cutaneous immune challenge by applying $20 \mu \mathrm{l}$ of 3-5\% 4-Ethoxymethylene-2-phenyl-2oxazolin-5-one (Sigma-Aldrich) in 1:2 acetone:olive oil. Peripheral LN (axillary, brachial, inguinal) were harvested at varying timepoints post inflammation for imaging. Complete Freund's adjuvant (CFA): Mice received a unilateral hock injection of $10 \mu \mathrm{l}$ Complete Freund's Adjuvant (Sigma-Aldrich), the popliteal LN were harvested three and half weeks later, and the inflamed nodes were compared to uninflamed control nodes with imaging.

Lineage tracing. For lineage tracing of CRP labeled in resting $\mathrm{LN}$ prior to immunization, reporter expression was induced in $A p \ln C r e E R \times R 26^{m T m G}$ mice by i.p. injection of $80 \mathrm{ug} / \mathrm{g}$ 4-hydroxytamoxifen (4-OHT; Sigma-Aldrich) either $24 \mathrm{~h}$ prior to sacrifice or immunization, or $76 \mathrm{~h}$ prior to sacrifice or immunization in separate experiments (see text and figure legends). Immunized mice were sacrificed at the indicated timepoints, and LN taken for imaging. Additional LN to control for leakiness of the reporter included LN from Apln ${ }^{\mathrm{CreER}} \times \mathrm{R}^{2} 6^{\mathrm{mTmG}}$ mice immunized in parallel without tamoxifen induction. For labeling and tracing of Apln ${ }^{C r e E R}$ expressing cells after initial immunization, reporter expression was induced in Apln CreER $\times$ R26 tdTomato mice the day after oxazolone skin painting, and lymph nodes were imaged $24 \mathrm{~h}$ later or after 11 days.

Statistical analysis. Statistical significance between two groups was calculated using a two-way ANOVA corrected with Tukey. The rule of three was applied to determine $95 \%$ confidence intervals for the enumeration of $\mathrm{ER}^{+}$cells. A likelihood ratio test was used for differential gene expression analysis assuming an underlying zero-inflated negative binomial distribution. $P$-values were adjusted for multiple comparisons by calculating the false discovery rate (FDR) and adjusted $p$-values $<0.001$ were considered significant.

Reporting summary. Further information on research design is available in the Nature Research Reporting Summary linked to this article.

\section{Data availability}

The authors declare that all data supporting the findings of this study are available within the article and its Supplementary Information files or from the corresponding author upon reasonable request. Raw single-cell RNAseq data have been deposited in the Gene Expression Ominibus (GEO) database under accession code: GSE140348. Processed single-cell data used in Supplementary Fig. 9 were obtained from the GEO (GSE108892 bone marrow endothelial cells; GSE106514 peripheral lymph node high endothelial cells) and the Tabula Muris consortium [https://registry.opendata.aws/tabula-muris-senis/]. Interactive web apps to visualize single-cell RNAseq data are available the PLN1 dataset [https://stanford.io/2qzJ8Hl] and the integrated dataset (PLN1, PLN2, and PLN3) [https://stanford.io/2WXR811].

\section{Code availability}

Code used in this study, including a customized implementation of the MAGIC algorithm, are available as part of the magicBatch package [https://github.com/kbrulois/ magicBatch].
Received: 12 March 2020; Accepted: 18 June 2020;

Published online: 30 July 2020

\section{References}

1. Anderson, A. O. \& Anderson, N. D. Studies on the structure and permeability of the microvasculature in normal rat lymph nodes. Am. J. Pathol. 80, 387-418 (1975).

2. Kumar, V. et al. Global lymphoid tissue remodeling during a viral infection is orchestrated by a B cell-lymphotoxin-dependent pathway. Blood 115, 4725-4733 (2010)

3. Chyou, S. et al. Coordinated regulation of lymph node vascular-stromal growth first by CD11c+ cells and then by T and B cells. J. Immunol. 187, 5558-5567 (2011).

4. Mondor, I. et al. Clonal proliferation and stochastic pruning orchestrate lymph node vasculature remodeling. Immunity 45, 877-888 (2016).

5. Rossi-Schneider, T. R., Verli, F. D., Marinho, S. A., Yurgel, L. S. \& Souza, M. A. L. D. Study of intussusceptive angiogenesis in inflammatory regional lymph nodes by scanning electron microscopy. Microsc. Res. Tech. 73, 14-19 (2010)

6. Lee, M. et al. Transcriptional programs of lymphoid tissue capillary and high endothelium reveal control mechanisms for lymphocyte homing. Nat. Immunol. 15, 982-995 (2014).

7. Girard, J.-P., Moussion, C. \& Förster, R. HEVs, lymphatics and homeostatic immune cell trafficking in lymph nodes. Nat. Rev. Immunol. 12, 762-773 (2012)

8. Vanlandewijck, M. et al. A molecular atlas of cell types and zonation in the brain vasculature. Nature 554, 475-480 (2018).

9. Potente, M. \& Mäkinen, T. Vascular heterogeneity and specialization in development and disease. Nat. Rev. Mol. Cell Biol. 18, 477-494 (2017).

10. Dermadi, D. et al. Exploration of cell development pathways through highdimensional single cell analysis in trajectory space. iScience 23, 100842 (2020).

11. Shin, D. \& Anderson, D. J. Isolation of arterial-specific genes by subtractive hybridization reveals molecular heterogeneity among arterial endothelial cells. Dev. Dyn. Publ. Am. Assoc. Anat. 233, 1589-1604 (2005).

12. Dekker, R. J. et al. Endothelial KLF2 links local arterial shear stress levels to the expression of vascular tone-regulating genes. Am. J. Pathol. 167, 609-618 (2005).

13. You, L.-R. et al. Suppression of Notch signalling by the COUP ${ }^{-}$TFII transcription factor regulates vein identity. Nature 435, 98-104 (2005)

14. Thiriot, A. et al. Differential DARC/ACKR1 expression distinguishes venular from non-venular endothelial cells in murine tissues. BMC Biol. 15, 45 (2017).

15. Sperandio, M., Gleissner, C. A. \& Ley, K. Glycosylation in immune cell trafficking. Immunol. Rev. 230, 97-113 (2009).

16. Weibel, E. R. Fifty years of Weibel-Palade bodies: the discovery and early history of an enigmatic organelle of endothelial cells1. J. Thromb. Haemost. 10, 979-984 (2012)

17. Jutila, M. A. et al. Inflammation-induced endothelial cell adhesion to lymphocytes, neutrophils, and monocytes. Role of homing receptors and other adhesion molecules. Transplantation 48, 727-731 (1989).

18. Bogoslowski, A., Butcher, E. C. \& Kubes, P. Neutrophils recruited through high endothelial venules of the lymph nodes via PNAd intercept disseminating Staphylococcus aureus. Proc. Natl Acad. Sci. USA 115 , 2449-2454 (2018)

19. Janatpour, M. J., Hudak, S., Sathe, M., Sedgwick, J. D. \& McEvoy, L. M. Tumor necrosis factor-dependent segmental control of MIG expression by high endothelial venules in inflamed lymph nodes regulates monocyte recruitment. J. Exp. Med. 194, 1375-1384 (2001).

20. Van Agtmael, T. et al. Col4al mutation in mice causes defects in vascular function and low blood pressure associated with reduced red blood cell volume. Hum. Mol. Genet. 19, 1119-1128 (2010).

21. Wang, L.-H. \& Baker, N. E. E proteins and ID proteins: helix-loop-helix partners in development and disease. Dev. Cell 35, 269-280 (2015).

22. Ling, F., Kang, B. \& Sun, X.-H. Id proteins: small molecules, mighty regulators. Curr. Top. Dev. Biol. 110, 189-216 (2014).

23. Khachigian, L. M. et al. Egr-1 is activated in endothelial cells exposed to fluid shear stress and interacts with a novel shear-stress-response element in the PDGF A-chain promoter. Arterioscler. Thromb. Vasc. Biol. 17, 2280-2286 (1997).

24. Nowicki, K. W. et al. Novel high-throughput in vitro model for identifying hemodynamic-induced inflammatory mediators of cerebral aneurysm formation. Hypertension 64, 1306-1313 (2014).

25. Vries, M. H. M. et al. CXCL1 promotes arteriogenesis through enhanced monocyte recruitment into the peri-collateral space. Angiogenesis 18, 163-171 (2015).

26. Bondareva, O. et al. Identification of atheroprone shear stress responsive regulatory elements in endothelial cells. Cardiovasc. Res. 115, 1487-1499 (2019)

27. Satija, R., Farrell, J. A., Gennert, D., Schier, A. F. \& Regev, A. Spatial reconstruction of single-cell gene expression data. Nat. Biotechnol. 33, 495-502 (2015). 
28. Zhou, Y., Williams, J., Smallwood, P. M. \& Nathans, J. Sox7, Sox17, and Sox18 cooperatively regulate vascular development in the mouse retina. PLoS ONE 10, $\mathrm{e} 0143650$ (2015).

29. Wright, D. E., Bowman, E. P., Wagers, A. J., Butcher, E. C. \& Weissman, I. L. Hematopoietic stem cells are uniquely selective in their migratory response to chemokines. J. Exp. Med. 195, 1145-1154 (2002)

30. Codega, P. et al. Prospective identification and purification of quiescent adult neural stem cells from their in vivo niche. Neuron 82, 545-559 (2014).

31. Fleischman, R. A. From white spots to stem cells: the role of the Kit receptor in mammalian development. Trends Genet. 9, 285-290 (1993).

32. Liu, Y. et al. Latexin inactivation enhances survival and long-term engraftment of hematopoietic stem cells and expands the entire hematopoietic system in mice. Stem Cell Rep. 8, 991-1004 (2017).

33. Holmfeldt, P. et al. Functional screen identifies regulators of murine hematopoietic stem cell repopulation. J. Exp. Med. 213, 433-449 (2016).

34. Kim, Y.-D. et al. The unique spliceosome signature of human pluripotent stem cells is mediated by SNRPA1, SNRPD1, and PNN. Stem Cell Res. 22, 43-53 (2017).

35. Chen, L.-L. \& Carmichael, G. G. Altered nuclear retention of mRNAs containing inverted repeats in human embryonic stem cells: functional role of a nuclear noncoding RNA. Mol. Cell 35, 467-478 (2009).

36. Teschendorff, A. E. \& Enver, T. Single-cell entropy for accurate estimation of differentiation potency from a cell's transcriptome. Nat. Commun. 8, 15599 (2017).

37. del Toro, R. et al. Identification and functional analysis of endothelial tip cellenriched genes. Blood 116, 4025-4033 (2010).

38. Strasser, G. A., Kaminker, J. S. \& Tessier-Lavigne, M. Microarray analysis of retinal endothelial tip cells identifies CXCR4 as a mediator of tip cell morphology and branching. Blood 115, 5102-5110 (2010).

39. Liu, Q. et al. Genetic targeting of sprouting angiogenesis using Apln-CreER. Nat. Commun. 6, 6020 (2015).

40. Lai, H. et al. B7-H3 modulates endothelial cell angiogenesis through the VEGF cytokine. Immunol. Res. 67, 202-211 (2019).

41. Robinson, S. P., Langan-Fahey, S. M., Johnson, D. A. \& Jordan, V. C. Metabolites, pharmacodynamics, and pharmacokinetics of tamoxifen in rats and mice compared to the breast cancer patient. Drug Metab. Dispos. 19, 36-43 (1991).

42. Chen, Q. et al. Apelin+ endothelial niche cells control hematopoiesis and mediate vascular regeneration after myeloablative injury. Cell Stem Cell 25, 768-783.e6 (2019).

43. Consortium, T. T. M. et al. A single cell transcriptomic atlas characterizes aging tissues in the mouse. bioRxiv https://doi.org/10.1101/661728 (2019).

44. Tikhonova, A. N. et al. The bone marrow microenvironment at single-cell resolution. Nature 569, 222-228 (2019).

45. Veerman, K., Tardiveau, C., Martins, F., Coudert, J. \& Girard, J.-P. Single-cell analysis reveals heterogeneity of high endothelial venules and different regulation of genes controlling lymphocyte entry to lymph nodes. Cell Rep. 26, 3116-3131.e5 (2019).

46. Goveia, J. et al. An integrated gene expression landscape profiling approach to identify lung tumor endothelial cell heterogeneity and angiogenic candidates. Cancer Cell 37, 21-36.e13 (2020).

47. Niethamer, T. K. et al. Defining the role of pulmonary endothelial cell heterogeneity in the response to acute lung injury. eLife 9, e53072 (2020).

48. Simons, M. \& Eichmann, A. Molecular controls of arterial morphogenesis. Circ. Res. 116, 1712-1724 (2015).

49. Yang, B. et al. Carbon dioxide permeability of aquaporin-1 measured in erythrocytes and lung of aquaporin-1 null mice and in reconstituted proteoliposomes. J. Biol. Chem. 275, 2686-2692 (2000).

50. Umetani, M. et al. Function of GATA transcription factors in induction of endothelial vascular cell adhesion molecule- 1 by tumor necrosis factor-alpha. Arterioscler. Thromb. Vasc. Biol. 21, 917-922 (2001).

51. $\mathrm{Xu}, \mathrm{J}$. et al. GPR68 senses flow and is essential for vascular physiology. Cell 173, 762-775.e16 (2018)

52. Kondeti, V. et al. Leukotriene D4 and prostaglandin E2 signals synergize and potentiate vascular inflammation in a mast cell-dependent manner through cysteinyl leukotriene receptor 1 and E-prostanoid receptor 3. J. Allergy Clin. Immunol. 137, 289-298 (2016)

53. Tsopanoglou, N. E. \& Maragoudakis, M. E. On the mechanism of thrombininduced angiogenesis. Potentiation of vascular endothelial growth factor activity on endothelial cells by up-regulation of its receptors. J. Biol. Chem. 274, 23969-23976 (1999).

54. Kertesz, N., Wu, J., Chen, T. H.-P., Sucov, H. M. \& Wu, H. The role of erythropoietin in regulating angiogenesis. Dev. Biol. 276, 101-110 (2004).

55. Xu, C. et al. Arteries are formed by vein-derived endothelial tip cells. Nat. Commun. 5, 5758 (2014).

56. Knaut, H., Werz, C., Geisler, R. \& Nüsslein-Volhard, C., Tübingen 2000 Screen Consortium. A zebrafish homologue of the chemokine receptor Cxcr4 is a germ-cell guidance receptor. Nature 421, 279-282 (2003).
57. Chang, G. H. K. et al. ARHGAP18: an endogenous inhibitor of angiogenesis, limiting tip formation and stabilizing junctions. Small GTPases 5, 1-15 (2014).

58. Lee, N. Y., Haney, J. C., Sogani, J. \& Blobe, G. C. Casein kinase $2 \beta$ as a novel enhancer of activin-like receptor-1 signaling. FASEB J. 23, 3712-3721 (2009).

59. Carmeliet, P. \& Jain, R. K. Molecular mechanisms and clinical applications of angiogenesis. Nature 473, 298-307 (2011).

60. Wakabayashi, T. et al. CD157 marks tissue-resident endothelial stem cells with homeostatic and regenerative properties. Cell Stem Cell 22, 384-397.e6 (2018).

61. Yu, Q. C., Song, W., Wang, D. \& Zeng, Y. A. Identification of blood vascular endothelial stem cells by the expression of protein $C$ receptor. Cell Res. 26, 1079-1098 (2016)

62. Fang, S., Wei, J., Pentinmikko, N., Leinonen, H. \& Salven, P. Generation of functional blood vessels from a single c-kit + adult vascular endothelial stem cell. PLoS Biol. 10, e1001407 (2012).

63. Kusumbe, A. P., Ramasamy, S. K. \& Adams, R. H. Coupling of angiogenesis and osteogenesis by a specific vessel subtype in bone. Nature 507, 323-328 (2014).

64. Tabula Muris, Consortium et al. Single-cell transcriptomics of 20 mouse organs creates a Tabula Muris. Nature 562, 367-372 (2018).

65. Gebala, V., Collins, R., Geudens, I., Phng, L.-K. \& Gerhardt, H. Blood flow drives lumen formation by inverse membrane blebbing during angiogenesis in vivo. Nat. Cell Biol. 18, 443-450 (2016).

66. Kalucka, J. et al. Single-cell transcriptome atlas of murine endothelial cells. Cell 180, 764-779.e20 (2020).

67. Blanco, R. \& Gerhardt, H. VEGF and Notch in tip and stalk cell selection. Cold Spring Harb. Perspect. Med. 3, a006569 (2013).

68. Pitulescu, M. E. et al. Dll4 and Notch signalling couples sprouting angiogenesis and artery formation. Nat. Cell Biol. 19, 915-927 (2017)

69. Valentin, G., Haas, P. \& Gilmour, D. The chemokine SDF1a coordinates tissue migration through the spatially restricted activation of Cxcr7 and Cxcr4b. Curr. Biol. 17, 1026-1031 (2007).

70. Tetteh, P. W. et al. Replacement of lost Lgr5-positive stem cells through plasticity of their enterocyte-lineage daughters. Cell Stem Cell 18, 203-213 (2016).

71. Xiang, M. et al. A single-cell transcriptional roadmap of the mouse and human lymph node lymphatic vasculature. Front. Cardiovasc. Med. 7, 52 (2020).

72. Lun, A. T. L., Bach, K. \& Marioni, J. C. Pooling across cells to normalize single-cell RNA sequencing data with many zero counts. Genome Biol. 17, 75 (2016).

73. van Dijk, D. et al. Recovering gene interactions from single-cell data using data diffusion. Cell 174, 716-729.e27 (2018).

74. Kowalczyk, M. S. et al. Single-cell RNA-seq reveals changes in cell cycle and differentiation programs upon aging of hematopoietic stem cells. Genome Res. 25, 1860-1872 (2015).

75. Haghverdi, L., Lun, A. T. L., Morgan, M. D. \& Marioni, J. C. Batch effects in single-cell RNA-sequencing data are corrected by matching mutual nearest neighbors. Nat. Biotechnol. 36, 421-427 (2018).

76. Gu, Z., Eils, R. \& Schlesner, M. Complex heatmaps reveal patterns and correlations in multidimensional genomic data. Bioinforma. Oxf. Engl. 32 2847-2849 (2016)

77. Tirosh, I. et al. Dissecting the multicellular ecosystem of metastatic melanoma by single-cell RNA-seq. Science 352, 189-196 (2016).

\section{Acknowledgements}

We thank Nicole Lazarus for technical advice, Dhananjay Wagh for single-cell sequencing, and Karen Hirschi for critical review. This work was supported by NIH grants R01 AI130471 and R37 AI047822 and award I01 BX-002919 from the Dept of Veterans Affairs to ECB, and by pilot awards under ITI Seed 122C158 and CCSB grant U54CA209971. K.B. was supported by NIH F32 CA200103; A.S. by the Mobility Plus fellowship from the Ministry of Science and Higher Education, Poland (1319/MOB/IV/ 2015/0); S.N. by the Swedish Society for Medical Research and Stanford Dean's Fellowship; M.R. by Swedish Research Council; and H.K. by an American Heart Association Fellowship; and J.K. by the Netherlands Organization for Scientific Research \# ALWOP.271. K.R. is supported by NIH grant RO1-HL128503 and is a New York Stem Cell Foundation-Robertson Investigator. Images in Figs. 1a, 1b, $3 \mathrm{cw}$ and $6 \mathrm{a}$ were created with BioRender.com.

\section{Author contributions}

K.B., A.R., A.S., S.N., A.B., H.K., M.R., and E.O. performed experiments. K.B. and S.N analyzed $10 \times$ data. K.B., S.N., A.R., A.S., J.P., and E.C.B. wrote and edited the manuscript H.K., B.Z., D.V., J.K., D.V., and R.H.A. contributed essential tools and advice. R.E.M., P.K., and K.H.A. provided critical input that drove results. E.C.B. supervised the study. 


\section{Competing interests}

The authors declare no competing interests.

\section{Additional information}

Supplementary information is available for this paper at https://doi.org/10.1038/s41467020-17291-5

Correspondence and requests for materials should be addressed to E.C.B.

Peer review information Nature Communications thanks Veronique Angeli and the other, anonymous, reviewer(s) for their contribution to the peer review of this work.

Reprints and permission information is available at http://www.nature.com/reprints

Publisher's note Springer Nature remains neutral with regard to jurisdictional claims in published maps and institutional affiliations (c) (i) Open Access This article is licensed under a Creative Commons Attribution 4.0 International License, which permits use, sharing, adaptation, distribution and reproduction in any medium or format, as long as you give appropriate credit to the original author(s) and the source, provide a link to the Creative Commons license, and indicate if changes were made. The images or other third party material in this article are included in the article's Creative Commons license, unless indicated otherwise in a credit line to the material. If material is not included in the article's Creative Commons license and your intended use is not permitted by statutory regulation or exceeds the permitted use, you will need to obtain permission directly from the copyright holder. To view a copy of this license, visit http://creativecommons.org/licenses/by/4.0/.

This is a U.S. government work and not under copyright protection in the U.S.; foreign copyright protection may apply 2020 